Pure and Applied Mathematics Quarterly

Volume 9, Number 4

(Special Issue: In memory of

Andrey Todorov, Part 1 of 3 )

$579-612,2013$

\title{
Quasi-Complete Intersection Homomorphisms
}

\author{
Luchezar L. Avramov, Inês Bonacho Dos Anjos Henriques \\ and Liana M. Şega
}

Dedicated to the memory of Andrey Todorov

\begin{abstract}
Extending a notion defined for surjective maps by Blanco, Majadas and Rodicio, we introduce and study a class of homomorphisms of commutative noetherian rings, which strictly contains the class of locally complete intersection homomorphisms while sharing many of its remarkable properties.
\end{abstract}

Keywords: Complete intersection ideals, Gorenstein ideals, Koszul homology, complete intersection rings, Gorenstein rings, Cohen-Macaulay rings, Poincaré series.

\section{INTRODUCTION}

This paper is concerned with those homomorphisms $\varphi: R \rightarrow S$ of commutative noetherian rings for which the homology functors $\mathrm{D}_{n}(S \mid R,-)$ of André [1] and Quillen [34] vanish for all $n \geq 3$. We call them quasi-complete intersection (or q.c.i.) homomorphisms, in view of the characterization of locally complete intersection (or l.c.i.) homomorphisms by the condition $\mathrm{D}_{n}(S \mid R,-)=0$ for $n \geq 2$; see $[34,1,8]$.

Received February 12, 2013.

2000 Mathematics Subject Classification. 13D02 (primary), 13A02, 13D07 (secondary).

Research partly supported by NSF grants DMS-0803082 and DMS-1103176 (LLA), NSF grant DMS-1101131 and Simons Foundation grant 20903 (LS). 
Quillen [34] conjectured that q.c.i. homomorphisms are the only maps that have finitely many non-vanishing André-Quillen homology functors. To devise a proof or to construct a counter-example, one needs to understand the structure of q.c.i. homomorphisms and/or to gain a detailed knowledge of their properties.

When $R$ is local J. Majadas Soto and A. Rodicio Garcia $[26,39,40]$ proved that surjective q.c.i. homomorphisms are quasi-Gorenstein in the sense of [9]. Rodicio conjectured that, up to faithfully flat base change, every q.c.i. homomorphism has a known form - it arises from a pair of embedded regular sequences; see [39].

Here the goal is to systematically investigate the properties of general q.c.i. homomorphisms. Our results show that they are remarkably similar to those of l.c.i. maps, which form a much smaller class. Following a template devised in [8]-[13] for studying homomorphisms of noetherian rings, we proceed in three stages.

The initial focus is on surjective homomorphisms of local rings. For such maps we study the q.c.i. property by using a description in terms of the Koszul homology of $\operatorname{Ker}(\varphi)$, due to Blanco, Majadas, and Rodicio [20]. Sections 1 through 6 contain, among other things, short new proofs of the theorems of Garcia and Soto; formulas for the changes of depth and (with restrictions) of Krull dimension, which raise questions concerning modules of finite Gorenstein dimension; descriptions, in closed form, of the changes in the homological behavior of the residue fields; examples of q.c.i. homomorphisms that do not have the form conjectured by Rodicio.

At a second stage, the results on surjective maps of local rings are extended to arbitrary local homomorphisms. This is done in Section 7 by utilizing Cohen factorizations of local homomorphisms, constructed in [13].

Finally, homomorphisms of noetherian rings are analyzed by patching the local results through vanishing theorems for an appropriate (co)homology theory. This is the content of Section 8, where the characterization of q.c.i. homomorphisms in terms of André-Quillen homology is used for the first time in this paper.

\section{Quasi-COMPlete InTERSECTION IDEALS}

Throughout this section $(R, \mathfrak{m}, k)$ denotes a local ring; in detail: $R$ is a commutative noetherian ring with unique maximal ideal $\mathfrak{m}$, and $k=R / \mathfrak{m}$. In addition, 
$I$ is an ideal of $R$ and we set $S=R / I$. We define quasi-complete intersection ideals, track their behavior under certain changes of rings, and provide examples.

Let $\boldsymbol{a}$ be a finite generating set of $I$ and $E$ the Koszul complex on $\boldsymbol{a}$. The homology $\mathrm{H}_{*}(E)$ has $\mathrm{H}_{0}(E)=S$ and a structure of graded-commutative $S$-algebra, inherited from the DG $R$-algebra $E$. Thus, there is a unique homomorphism

$$
\lambda_{*}^{S}: \Lambda_{*}^{S} \mathrm{H}_{1}(E) \longrightarrow \mathrm{H}_{*}(E)
$$

of graded $S$-algebras with $\lambda_{1}^{S}=\mathrm{id}^{\mathrm{H}_{1}(E)}$, where $\Lambda_{*}^{S}$ is the exterior algebra functor.

Note that $\lambda_{*}^{S}$ is bijective if (and only if) there exists some isomorphism of graded $S$-algebras $\lambda: \mathrm{H}_{*}(E) \stackrel{\cong}{\rightarrow} \Lambda_{*}^{S} \mathrm{H}_{1}(E)$. Indeed, when this is the case the composed map

$$
\Lambda_{*}^{S} \mathrm{H}_{1}(E) \stackrel{\Lambda_{*}^{S}\left(\lambda_{1}\right)}{\longrightarrow} \Lambda_{*}^{S} \mathrm{H}_{1}(E) \stackrel{\lambda^{-1}}{\longrightarrow} \mathrm{H}_{*}(E)
$$

is an isomorphism, and it is equal to $\lambda_{*}^{S}$ because both maps restrict to $\mathrm{id}^{\mathrm{H}_{1}(E)}$.

1.1. We say that $I$ is quasi-complete intersection if $\mathrm{H}_{1}(E)$ is free over $S$ and $\lambda_{*}^{S}$ is bijective; this property does not depend on the choice of $\boldsymbol{a}$, see [21, 1.6.21].

To the best of our knowledge, the ideals defined above first appeared, with no denomination, in Rodicio's paper [36]; in his joint paper [19] with Blanco and Majadas, their defining property is called free exterior Koszul homology.

Recall that grade $R$ denotes the maximal length of an $R$-regular sequence in $I$, and that this number is equal to the least integer $n$ with $\operatorname{Ext}_{R}^{n}(S, R) \neq 0$.

Lemma 1.2. If I is quasi-complete intersection, then

$$
\operatorname{grade}_{R} S=\operatorname{rank}_{R} E_{1}-\operatorname{rank}_{S} \mathrm{H}_{1}(E) .
$$

Proof. The grade-sensitivity of $E$, see $[21,1.6 .17(\mathrm{~b})]$, yields

$$
\operatorname{rank}_{R} E_{1}-\operatorname{grade}_{R} S=\max \left\{n \mid \mathrm{H}_{n}(E) \neq 0\right\} .
$$

As $\mathrm{H}_{*}(E)$ is isomorphic to $\Lambda_{*}^{S} \mathrm{H}_{1}(E)$, the right-hand side equals $\operatorname{rank}_{S} \mathrm{H}_{1}(E)$.

Quasi-complete intersection ideals are stable under certain base changes.

Lemma 1.3. Let $R^{\prime}$ be a local ring and $\rho: R \rightarrow R^{\prime}$ a flat homomorphism of rings.

When $I R^{\prime} \neq R^{\prime}$ holds, if I is quasi-complete intersection, then so is $I R^{\prime}$.

When $\rho$ is faithfully flat, if $I R^{\prime}$ is quasi-complete intersection, then so is $I$. 
Proof. Note that $R^{\prime} \otimes_{R} E$ is the Koszul complex on the generating set $\rho(\boldsymbol{a})$ of $I R^{\prime}$. As $R^{\prime}$ is a flat $R$-module, there is a natural isomorphism of graded algebras $\mathrm{H}_{*}\left(R^{\prime} \otimes_{R} E\right) \cong R^{\prime} \otimes_{R} \mathrm{H}_{*}(E)$, whence the assertions follow by standard arguments.

Lemma 1.4. Let $\boldsymbol{b}$ be an $R$-regular sequence contained in $I$.

The ideal $I$ of $R$ is quasi-complete intersection if and only if the ideal $\bar{I}=I /(\boldsymbol{b})$ of $\bar{R}=R /(\boldsymbol{b})$ is quasi-complete intersection.

Proof. By induction, we may assume $\boldsymbol{b}=b$. Let $E$ be the Koszul complex on a generating set $\left\{a_{1}, \ldots, a_{c}\right\}$ of $I$ with $a_{1}=b$. Pick $v_{1}, \ldots, v_{c}$ in $E_{1}$ with $\partial\left(v_{i}\right)=a_{i}$ for $i=1, \ldots, c$, set $v=v_{1}$, and note that $J:=(b, v) E$ is a DG ideal. Each $y \in J$ can be written as $y=b e+v f$ with $e, f \in E^{\prime}$, where $E^{\prime}$ is the DG $R$-subalgebra of $E$, generated by $v_{2}, \ldots, v_{c}$. Now $\partial(y)=0$ implies $b(\partial(e)+f)=0$. As $b$ is regular, this gives $f=-\partial(e)$, hence $y=\partial(v e)$. We proved $\mathrm{H}_{*}(J)=0$, so $E \rightarrow E / J$ induces $\mathrm{H}_{*}(E) \cong \mathrm{H}_{*}(E / J)$ as graded $S$-algebras. It remains to note that $E / J$ is isomorphic to the Koszul complex on $\boldsymbol{a}=\left\{a_{2}+(\boldsymbol{b}), \ldots, a_{c}+(\boldsymbol{b})\right\} \subseteq \bar{R}$, and $\bar{I}=(\boldsymbol{a})$.

In our study of quasi-complete intersection ideals a fundamental role is played by a classical construction of Tate [41]; see also [28, §1.1], [7, §6].

Construction 1.5. Let $\boldsymbol{a}=\left\{a_{1}, \ldots, a_{c}\right\}$ be a generating set of $I$ and $E$ the Koszul complex on $\boldsymbol{a}$. Fix a basis $\boldsymbol{v}=\left\{v_{1}, \ldots, v_{c}\right\}$ of $E_{1}$ with $\partial\left(v_{i}\right)=a_{i}$ for $1 \leq i \leq c$ and a set of cycles $\boldsymbol{z}=\left\{z_{1}, \ldots, z_{h}\right\}$ whose classes minimally generate $\mathrm{H}_{1}(E)$.

Let $W$ be a graded $R$-module that has a basis $\boldsymbol{w}=\left\{w_{1}, \ldots, w_{h}\right\}$ of elements of degree 2 , and let $\Gamma_{*}^{R} W$ be the divided powers algebra on $W$. The products $w_{1}^{\left(j_{1}\right)} \cdots w_{h}^{\left(j_{h}\right)}$ with $j_{i} \geq 0$ and $j_{1}+\cdots+j_{h}=p$ form an $R$-basis of $\Gamma_{p}^{R} W$.

The Tate complex on $E$ and $\boldsymbol{z}$ is the complex $F$ of free $R$-modules with

$$
\begin{aligned}
F_{n} & =\bigoplus_{2 p+q=n} \Gamma_{p}^{R} W \otimes_{R} E_{q} . \\
\partial_{n}^{F}\left(w_{1}^{\left(j_{1}\right)} \cdots w_{h}^{\left(j_{h}\right)} \otimes e\right)= & w_{1}^{\left(j_{1}\right)} \cdots w_{h}^{\left(j_{h}\right)} \otimes \partial^{E}(e) \\
& \quad+\sum_{i=1}^{h} w_{1}^{\left(j_{1}\right)} \cdots w_{i}^{\left(j_{i}-1\right)} \cdots w_{h}^{\left(j_{h}\right)} \otimes z_{i} e .
\end{aligned}
$$


It is clear from the construction that $\mathrm{H}_{0}(F)=S$ holds.

1.6. Let $F$ be the complex in Construction 1.5. From [41, Theorems 4] one gets:

If $I$ is quasi-complete intersection, then $F$ is a free resolution of the $R$-module $S$. If $\boldsymbol{a}$ generates $I$ minimally, then $F$ is minimal: each $z_{i}$ then is a syzygy in the free cover $E_{1} \rightarrow I$, whence $z_{i} \in \mathfrak{m} E_{1}$, so $\partial(F) \subseteq \mathfrak{m} F$ holds by (1.5.2).

We finish the section with descriptions of quasi-complete intersection ideals in terms of the homology functors $\mathrm{D}_{n}(S \mid R,-)$ of André [1] and Quillen [34]. Until the last section, these are used solely for comparisons with existing results or arguments.

1.7. The ideal $I$ is quasi-complete intersection if and only if $\mathrm{D}_{n}(S \mid R,-)=0$ holds for $n \geq 3$, if and only if $\mathrm{D}_{n}(S \mid R, k)=0$ holds for $n \geq 3$.

The first equivalence is due to Blanco, Majadas, and Rodicio and appears in [20, Corollary $\left.3^{\prime}\right]$; the proofs of similar statements in [36] and [19] are incomplete, see $[20$, pp. 126-127]. The second equivalence follows from $[1,4.57]$.

\section{Between Complete intersection And Gorenstein}

In this section $I$ is an ideal in a local $\operatorname{ring}(R, \mathfrak{m}, k)$. We compare the quasicomplete intersection property and other properties of ideals, which we recall next.

2.1. Recall that $I$ is said to be complete intersection if it has a generating set $\boldsymbol{a}$ satisfying the following equivalent conditions: (i) $\boldsymbol{a}$ is an $R$-regular sequence; (ii) $\mathrm{H}_{1}(E)=0$; (iii) $\mathrm{H}_{n}(E)=0$ for all $n \geq 1$. Thus, one evidently has:

Every complete intersection ideal is quasi-complete intersection.

The ideal $\mathfrak{m}$ is complete intersection if and only if $R$ is regular; see [21, 2.2.5].

2.2. By Cohen's Structure Theorem, the m-adic-completion of $R$ has a presentation $\widehat{R} \cong Q / J$, with $Q$ a regular local ring. The ring $R$ is said to be complete intersection if in some Cohen presentation of $\widehat{R}$ the ideal $J$ is generated by a regular sequence; this property is independent of the presentation, see [27, 19.3.2] or $[21,2.3 .3]$.

The ideal $\mathfrak{m}$ is quasi-complete intersection if and only if the ring $R$ is complete intersection; this is due to Assmus, [4, 2.7], see also [21, 2.3.11]. 
2.3. A quasi-deformation is a pair $R \rightarrow R^{\prime} \leftarrow Q$ of homomorphisms of local rings, where $R \rightarrow R^{\prime}$ is faithfully flat and $R^{\prime} \leftarrow Q$ is surjective with kernel generated by a $Q$-regular sequence. By definition, the $C I$-dimension of an $R$-module $M$, denoted CI- $\operatorname{dim}_{R} M$, is finite if $\operatorname{pd}_{Q}\left(R^{\prime} \otimes_{R} M\right)$ is finite for some quasi-deformation; see $[15]$.

If $R$ is complete intersection, then CI- $\operatorname{dim}_{R} M$ is finite for each $M$.

If CI- $\operatorname{dim}_{R} \mathfrak{m}$ is finite, then $R$ is complete intersection; see $[15,1.3,1.9]$.

2.4. We say that $I$ is quasi-Gorenstein if $\operatorname{Ext}_{R}^{\operatorname{grade}_{R} S}(S, R) \cong S$ and $\operatorname{Ext}_{R}^{n}(S, R)=$ 0 for $n>\operatorname{grade}_{R} S$; thus, $\mathfrak{m}$ is quasi-Gorensten if and only if $R$ is a Gorenstein ring.

By $[17,2.3]$, the ideal $I$ is Gorenstein if and only if the homomorphism $R \rightarrow$ $R / I$ is quasi-Gorenstein in the sense of [11]. In particular, $[11,6.5,7.4,7.5]$ yield

$$
\operatorname{grade}_{R} S=\operatorname{depth} R-\operatorname{depth} S \text {. }
$$

A short proof of this equality, following [26, Corollary 5], proceeds as follows:

Set $d=\operatorname{depth} S$ and $g=\operatorname{grade}_{R} S . \operatorname{As}_{\operatorname{Ext}_{R}}^{g}(S, R) \cong S$, the spectral sequence

$$
\mathrm{E}_{2}^{p, q}=\operatorname{Ext}_{S}^{p}\left(k, \operatorname{Ext}_{R}^{q}(S, R)\right) \Longrightarrow \operatorname{Ext}_{R}^{p+q}(k, R)
$$

yields $\operatorname{Ext}_{R}^{p}(k, R)=0$ for $p<d+g$, and $\operatorname{Ext}_{R}^{d+g}(k, R) \cong \operatorname{Ext}_{S}^{d}(k, S) \neq 0$.

We present new, direct proofs of two key results of Garcia and Soto, comparing the quasi-complete intersection condition with other properties: The implication (3) in the next theorem is [39, Proposition 12] and (4) is obtained in [26, Remark 8]. 
Theorem 2.5. For an ideal I of a local ring $R$, the ring $S=R / I$, and the Koszul complex $E$ on some set of generators for I the following implications hold $I$ is complete intersection $\stackrel{(1)}{\Longleftrightarrow} \begin{gathered}\text { is quasi-complete intersection } \\ \text { and } \operatorname{pd}_{R} S \text { is finite }\end{gathered}$

I is quasi-complete intersection

(3) $\mathrm{H}_{1}(E)$ is free over $S$ and $\mathrm{CI}-\operatorname{dim}_{R} S$ is finite

\author{
(4) $\Downarrow$ \\ I is quasi-Gorenstein
}

Remark 2.6. The simple implications in the theorem are irreversible: For (2), apply 2.1 and 2.2 with $R=k[x] /\left(x^{2}\right)$ and $I=\mathfrak{m}$. For (4), apply 2.2 and 2.4 with $R=k[x, y, z] /\left(x^{2}-y^{2}, y^{2}-z^{2}, x y, x z, y z\right)$ and $I=\mathfrak{m}$. For (3), see Theorem 3.5.

To prove (3) we use a lemma that can be extracted from the argument for [39, Proposition 12], which uses André-Quillen homology. Here is a short direct proof.

Lemma 2.7. (Notation as in Theorem 2.5) When CI- $\operatorname{dim}_{R} S$ is finite and $\mathrm{H}_{1}(E)$ is free over $S$ there exist a local ring $Q$, complete intersection ideals $J \subseteq I^{\prime}$ of $Q$, and a flat local homomorphism $R \rightarrow Q / J$ such that $I(Q / J)=I^{\prime} / J$.

Proof. Let $R \rightarrow R^{\prime} \leftarrow Q$ be a quasi-deformation with $\operatorname{pd}_{Q}\left(R^{\prime} \otimes_{R} S\right)$ finite, set $S^{\prime}=R^{\prime} \otimes_{R} S$ and $J=\operatorname{Ker}\left(Q \rightarrow R^{\prime}\right)$; we may assume $R^{\prime}=Q / J$. Let $E$ be the Koszul complex on a set $\left\{a_{1}, \ldots, a_{c}\right\}$ of generators of $I$. Choose in $Q$ a set $\boldsymbol{a}^{\prime}=\left\{a_{1}^{\prime}, \ldots, a_{c}^{\prime}\right\}$, such that $a_{i}^{\prime}$ maps to the image of $a_{i}$ in $R^{\prime}$, and let $E^{\prime}$ be the Koszul complex on $\boldsymbol{a}^{\prime}$. Let $B$ the Koszul complex on a $Q$-regular set $\boldsymbol{b}$ generating $J$. The quasi-isomorphism $B \rightarrow R^{\prime}$ induces a quasi-isomorphism $B \otimes_{Q} E^{\prime} \rightarrow R^{\prime} \otimes_{Q} E^{\prime}$. It gives the first isomorphism in the following string, where the second one is induced by $R^{\prime} \otimes_{Q} E^{\prime} \cong R^{\prime} \otimes_{R} E$, and the third one is due to the flatness of $R^{\prime}$ over $R$ :

$$
\mathrm{H}_{1}\left(B \otimes_{Q} E^{\prime}\right) \cong \mathrm{H}_{1}\left(R^{\prime} \otimes_{Q} E^{\prime}\right) \cong \mathrm{H}_{1}\left(R^{\prime} \otimes_{R} E\right) \cong R^{\prime} \otimes_{R} \mathrm{H}_{1}(E) \cong S^{\prime} \otimes_{S} \mathrm{H}_{1}(E)
$$

Thus, $\mathrm{H}_{1}\left(B \otimes_{Q} E^{\prime}\right)$ is free over $S^{\prime}$. As $B \otimes_{Q} E^{\prime}$ is the Koszul complex on $\boldsymbol{b} \sqcup \boldsymbol{a}^{\prime}$, Gulliksen $[28,1.4 .9]$ shows that the ideal $I^{\prime}=Q\left(\boldsymbol{b} \sqcup \boldsymbol{a}^{\prime}\right)$ is complete intersection. The choices made yield $I^{\prime} / J=I R^{\prime} / J=I(Q / J)$, as desired. 
In [26] the implication (4) in Theorem 2.5 is derived from a result concerning algebras with Poincaré duality. Our proof uses the DG E-module structure of Tate complexes. We describe some notions used to handle it.

2.8. Let $X$ be a complex of $R$-modules. We let $|x|=n$ stand for $x \in X_{n}$.

The complex $\Sigma X$ has $(\Sigma X)_{n}=X_{n-1}$ and $\partial_{n}^{\Sigma X}=-\partial_{n-1}^{X}$; for $n \in \mathbb{Z}$; for $x \in X_{n-1}$ we let $\varsigma(x)$ denote the element $x \in(\Sigma X)_{n}$. When $X$ is a DG $E$ module, so is $\Sigma X$, with action of $E$ given by $e \varsigma(x)=(-1)^{i} \varsigma(e x)$ for $e \in E_{i}$.

The complex $X^{\vee}$ is defined by $\left(X^{\vee}\right)_{n}=\operatorname{Hom}_{R}\left(X_{-n}, R\right)$ and $\left(\partial^{X^{\vee}}(\chi)\right)(x)=$ $(-1)^{n+1} \chi \partial^{X}(x)$ for $\chi \in\left(X^{\vee}\right)_{n}, x \in X_{-n+1}$, and $n \in \mathbb{Z}$. When $X$ is a DG $E$-module, so is $X^{\vee}$, with action of $E$ given by $(e \cdot \chi)(x)=(-1)^{|i||\chi|} \chi(e x)$ for $e \in E_{i}$.

Proof of Theorem 2.5. (1) If $I$ is quasi-complete intersection and $\operatorname{pd}_{R} S$ is finite, then $F_{n}=0$ for $n \gg 0$ in the minimal resolution $F$ of 1.6. This forces $W=0$, hence $\mathrm{H}_{1}(E)=0$, so $I$ is complete intersection. The converse is clear.

(2) This implication holds because finite projective dimension implies finite CI-dimension, as testified by the quasi-deformation $R \stackrel{=}{\longrightarrow} \stackrel{=}{\longleftarrow}$.

(3) This follows directly from Lemmas 2.7 and 1.3.

(4) We use the notation from Construction 1.5. Let $\boldsymbol{\omega}=\left\{\omega_{1}, \ldots, \omega_{h}\right\}$ be the basis of $W^{\vee}$, dual to $\boldsymbol{w}$, and $\mathrm{S}_{*}^{R}\left(W^{\vee}\right)$ the symmetric algebra of $W^{\vee}$. Standard isomorphisms of $R$-modules, $\left(\Gamma_{p}^{R} W\right)^{\vee} \cong \mathrm{S}_{p}^{R}\left(W^{\vee}\right)$, take the elements of the basis of $\left(\Gamma_{p}^{R} W\right)^{\vee}$, dual to the basis of $\Gamma_{p}^{R} W$ described in Construction 1.5, to the corresponding products $\omega_{1}^{j_{1}} \cdots \omega_{h}^{j_{h}}$. Thus, we have

$$
\begin{gathered}
\left(F^{\vee}\right)_{n}=\bigoplus_{2 p+q=n} \mathrm{~S}_{-p}^{R}\left(W^{\vee}\right) \otimes_{R}\left(E^{\vee}\right)_{q} . \\
\partial^{F^{\vee}}(\omega \otimes \epsilon)=\omega \otimes \partial^{E^{\vee}}(\epsilon)+\sum_{i=1}^{h} \omega_{i} \omega \otimes z_{i} \cdot \epsilon .
\end{gathered}
$$

A decreasing filtration of $F^{\vee}$ is given by the $R$-linear spans of the sets

$$
\left\{\left(\omega_{1}^{j_{1}} \cdots \omega_{h}^{j_{h}} \otimes \epsilon\right) \in F^{\vee} \mid j_{i} \geq 0, j_{1}+\cdots+j_{h} \geq-p, \epsilon \in E^{\vee}\right\}
$$

for $p \leq 0$. The resulting spectral sequence $\mathrm{E}_{p, q}^{r} \Longrightarrow \mathrm{H}_{p+q}\left(F^{\vee}\right)$ starts with

$$
\mathrm{E}_{p, q}^{0}=\mathrm{S}_{-p}^{R}\left(W^{\vee}\right) \otimes_{R}\left(E^{\vee}\right)_{q-p} \quad \text { and } \quad \mathrm{d}_{p, q}^{0}=\mathrm{S}_{-p}^{R}\left(W^{\vee}\right) \otimes_{R} \partial_{q-p}^{E^{\vee}} .
$$


Let $U$ be an $R$-module with basis $\boldsymbol{u}=\left\{u_{1}, \ldots, u_{h}\right\}$. The hypothesis on $I$ yields an isomorphism $S \otimes_{R} \Lambda_{*}^{R} U \cong \mathrm{H}_{*}(E)$ of graded $R$-algebras sending $u_{i}$ to the class of $z_{i}$ for $i=1, \ldots, h$. Let $\tau^{u} \in\left(\Lambda_{*}^{R} U\right)_{-h}^{\vee}$ be the $R$-linear map with $\tau^{\boldsymbol{u}}\left(u_{1} \cdots u_{h}\right)=1$; the map $u \mapsto u \cdot \tau^{\boldsymbol{u}}$ is an isomorphism $\Lambda_{*}^{R} U \cong \Sigma^{h}\left(\Lambda_{*}^{R} U\right)^{\vee}$ of graded $\Lambda_{*}^{R} U$-modules. Similarly, the $R$-linear map $\tau^{\boldsymbol{v}} \in\left(E^{\vee}\right)_{-c}$, defined by $\tau^{\boldsymbol{v}}\left(v_{1} \cdots v_{c}\right)=1$, yields an isomorphism of DG $E$-modules $E \cong \Sigma^{-c}\left(E^{\vee}\right)$, given by $e \mapsto e \cdot \tau^{v}$. Thus, we get

$$
\mathrm{H}_{q-p}\left(E^{\vee}\right) \cong \mathrm{H}_{q-p+c}(E) \cong S \otimes_{R} \Lambda_{q-p+c}^{R} U \cong S \otimes_{R}\left(\Lambda_{*}^{R} U\right)_{q-p+c-h}^{\vee}
$$

as $R$-modules. Now (2.9.3) and (2.9.4) yield

$$
\begin{aligned}
\mathrm{E}_{p, q}^{1}=\mathrm{H}_{q}\left(\mathrm{E}_{p, \bullet}^{0}\right) & \cong \mathrm{S}_{-p}^{R}\left(W^{\vee}\right) \otimes_{R} \mathrm{H}_{q-p}\left(E^{\vee}\right) \\
& \cong S \otimes_{R}\left(\mathrm{~S}_{-p}^{R}\left(W^{\vee}\right) \otimes_{R}\left(\Lambda_{*}^{R} U\right)_{q-p+c-h}^{\vee}\right) \\
& \cong S \otimes_{R}\left(\Gamma_{-p}^{R}(W) \otimes_{R} \Lambda_{p-q+(h-c)}^{R} U\right)^{\vee} .
\end{aligned}
$$

Let $C$ be the complex with $C_{n}=\bigoplus_{2 p+q=n} \mathrm{E}_{p, q}^{1}$ and $\partial_{n}^{C}=\bigoplus_{2 p+q=n} \mathrm{~d}_{p, q}^{1}$. Let $G$ be the Tate complex on the Koszul complex $\Lambda_{*}^{R} U$ with zero differentials and the set of cycles $\boldsymbol{u}$. Formulas (2.9.2) and (1.5.2) show that the maps above produce an isomorphism of complexes $C \cong S \otimes_{R}\left(\Sigma^{c-h} G\right)^{\vee}$. By 1.6, $G$ is an $R$-free resolution of $R$, so $G$ is homotopically equivalent to $R$. Consequently, $\left(\Sigma^{c-h} G\right)^{\vee}$ is homotopically equivalent to $\Sigma^{h-c} R$, whence $\mathrm{H}_{n}(C)=0$ for $n \neq h-c$, and $\mathrm{H}_{h-c}(C) \cong S$. We have $C_{h-c}=\mathrm{E}_{0, h-c}^{1}$ so the computation of $\mathrm{H}_{*}(C)$ yields

$$
\mathrm{E}_{p, q}^{2}=\mathrm{H}_{p}\left(\mathrm{E}_{\bullet, q}^{1}\right) \cong \begin{cases}S & \text { for }(p, q)=(0, h-c), \\ 0 & \text { otherwise } .\end{cases}
$$

The convergence of the spectral sequence implies $\mathrm{H}_{h-c}\left(F^{\vee}\right) \cong S$ and $\mathrm{H}_{n}\left(F^{\vee}\right)=$ 0 for $n \neq h-c$. One has $\mathrm{H}_{n}\left(F^{\vee}\right) \cong \operatorname{Ext}_{R}^{-n}(S, R)$, see 1.6, hence $\operatorname{Ext}_{R}^{c-h}(S, R) \cong S$ and $\operatorname{Ext}_{R}^{n}(S, R)=0$ for $n \neq c-h$, so $I$ is quasi-Gorenstein.

\section{ExACT IDEALS}

In this section $(R, \mathfrak{m}, k)$ denotes a local ring. We first discuss principal quasicomplete intersection ideals. They are easy to identify, are amenable to explicit computations, and have turned up in abundance in recent studies. Exact ideals are defined in terms of principal quasi-complete intersection ideals by analogy with the way that complete intersection ideals are formed from regular elements. 
3.1. Let $N$ be an $R$-module. We say that an element $a$ of $R$ is an exact zerodivisor on $N$ if there exists an element $b$ of $R$ that satisfies

$$
(0: a)_{N}=b N \neq 0 \quad \text { and } \quad a N=(0: b)_{N} \neq 0 .
$$

This implies that the following sequence is exact:

$$
\cdots \rightarrow N \stackrel{b}{\rightarrow} N \stackrel{a}{\rightarrow} N \stackrel{b}{\rightarrow} N \stackrel{a}{\rightarrow} N \rightarrow \cdots
$$

Thus, $(a, b)$ is an $N$-exact pair in the sense of Kiełpiński, Simson, and Tyc [30, $1.1]$, and $b$ also is an exact zero-divisor on $N$.

Following [29,1.2], when $N=R$ we call $a$ an exact zero-divisor. In this case conditions in (3.1.1) are equivalent to the following simpler requirement:

$$
R \neq(0: a)_{R} \cong R /(a) \neq 0 .
$$

Indeed, it implies $(0: a)_{R}=(b) \neq 0$ for some $b$ in $R$; the map $R \rightarrow(b)$ with $1 \mapsto b$ and its composition with $(b) \cong R /(a)$ have the same kernel, hence $(0: b)_{R}=(a)$.

We say that an element $a$ of $R$ is exact if it is $R$-regular or an exact zero-divisor. This holds if and only if $(a)$ is quasi-complete intersection: The Koszul complex $E$ on $\{a\}$ has $E_{\geqslant 2}=0$ and $\mathrm{H}_{1}(E)=(0: a)_{R}$, so $\mathrm{H}_{1}(E)$ is free over $R /(a)$ and $\lambda_{*}^{R /(a)}$ in (1.0.1) is bijective if and only if $(0: a)_{R}=0$ or $(0: a)_{R} \cong R /(a)$.

In some cases, only half of the conditions in (3.1.1) need verification.

Lemma 3.2. Let $N$ be an $R$-module of finite length.

If $(0: b)_{N}=a N$ holds for some elements $a, b \in R$, then one has $(0: a)_{N}=b N$.

Proof. From $a(b N)=b(a N)=b(0: b)_{N}=0$ one gets $(0: a)_{N} \supseteq b N$. A length count, using this inclusion and the composition $N / a N=N /(0: b)_{N} \cong b N$, gives

$$
\ell(N)-\ell(a N)=\ell\left((0: a)_{N}\right) \geq \ell(b N)=\ell(N / a N)=\ell(N)-\ell(a N) .
$$

These relations imply $\ell\left((0: a)_{N}\right)=\ell(b N)$, hence $(0: a)_{N}=b N$.

For Cohen-Macaulay rings the situation is only slightly more complicated.

Proposition 3.3. For an element a of $\mathfrak{m}$ the following conditions are equivalent.

(i) $a$ is an exact zero-divisor and $R$ is Cohen-Macaulay.

(ii) $(0: a)_{R}=(b) \neq R$ for some $b$ in $R$, and $\operatorname{depth} R /(a) \geq \operatorname{dim} R$. 
(iii) $(0: a)_{R}=(b) \neq R$ for some $b$ in $R$, and $R /(a)$ is Cohen-Macaulay with $\operatorname{dim} R /(a)=\operatorname{dim} R$.

Proof. (i) $\Longrightarrow$ (ii). Using (2.4.1), we get $\operatorname{depth} R /(a)=\operatorname{depth} R=\operatorname{dim} R$.

(ii) $\Longrightarrow$ (iii). Use the inequalities $\operatorname{dim} R \geq \operatorname{dim} R /(a) \geq \operatorname{depth} R /(a)$.

(iii) $\Longrightarrow$ (i). Set $K=(0: b)_{R} /(a)$ and pick $\mathfrak{p} \in \operatorname{Ass}_{R}(K)$. From $K \subseteq R /(a)$ we get $\mathfrak{p} \in \operatorname{Ass}_{R} R /(a)$. The Cohen-Macaulayness of $R /(a)$ implies that $\mathfrak{p}$ is minimal in $\operatorname{Supp}_{R} R /(a)$ and satisfies $\operatorname{dim}(R / \mathfrak{p})=\operatorname{dim} R /(a)$. We have $\operatorname{dim} R /(a)=$ $\operatorname{dim} R$, so $\mathfrak{p}$ is minimal in Spec $R$, hence the $\operatorname{ring} R_{\mathfrak{p}}$ is artinian. As one has $(0:(a / 1))_{R_{\mathfrak{p}}}=(b / 1) R_{\mathfrak{p}}$, Lemma 3.2 yields $(a / 1) R_{\mathfrak{p}}=(0:(b / 1))_{R_{\mathfrak{p}}}$; that is, $K_{\mathfrak{p}}=0$. Since $\mathfrak{p}$ can be chosen arbitrarily in $\operatorname{Ass}_{R}(K)$, we conclude that $K$ is equal to zero.

Thus, $a$ is an exact zero-divisor. Now (2.4.1) and the hypotheses yield $\operatorname{depth} R=\operatorname{depth} R /(a)=\operatorname{dim} R /(a)=\operatorname{dim} R$.

Remark 3.4. The implication (iii) $\Longrightarrow$ (i) may fail when $\operatorname{dim} R /(a) \neq \operatorname{dim} R$.

Indeed, set $R=k[x, y] /\left(x y, y^{2}\right)$, and let $a$ and $b$ denote the images in $R$ of $x$ and $y$, respectively. The equality $(0: a)_{R}=(b)$ shows that $(0: a)_{R}$ is principal, and the isomorphism $R /(a) \cong k[y] /\left(y^{2}\right)$ that $R /(a)$ is Cohen-Macaulay. However, $R$ is not Cohen-Macaulay; neither is $a$ an exact zero-divisor, as $(0: b)_{R}=(a, b) \neq(a)$.

Now we settle, in the negative, a conjecture of Rodicio; see [39, Conjecture 11].

Theorem 3.5. If $k$ is a field of characteristic different from 2, then in the ring

$$
R=\frac{k[w, x, y, z]}{\left(w^{2}, w x-y^{2}, w y-x z, w z, x^{2}+y z, x y, z^{2}\right)}
$$

the ideal $x R$ is quasi-complete intersection and has infinite CI-dimension.

Proof. Let $a$ and $b$ denote the images in $R$ of $x$ and $y$, respectively; thus, $x R=(a)$. A simple calculation yields $(0: a)_{R}=(b)$ and $(0: b)_{R}=(a)$, so $a$ is an exact zero-divisor, and hence $(a)$ is a quasi-complete intersection ideal; see 3.1.

Assume CI- $\operatorname{dim}_{R} I$ is finite. Yoneda products then turn $\operatorname{Ext}_{R}^{*}(R /(a), k)$ into a finite graded module that is finite over a $k$-subalgebra of $\operatorname{Ext}_{R}^{*}(k, k)$, generated by central elements of degree 2 ; see $[18,5.3]$. On the other hand, in $[14$, p. 4$]$ it is shown that $\operatorname{Ext}_{R}^{2}(k, k)$ contains no non-zero central element of $\operatorname{Ext}_{R}^{*}(k, k)$, hence 
$\operatorname{Ext}_{R}^{n}(R /(a), k)=0$ for all $n \gg 0$. This is impossible, since (3.1.2) with $N=R$ gives a free resolution of $R /(a)$ that yields $\operatorname{Ext}_{R}^{n}(R /(a), k) \cong k$ for $n \geq 0$.

In $[30,1.1]$ exact sequences of pairs are defined by analogy with regular sequences of elements. Both notions are subsumed into the next concept.

3.6. A sequence $a_{1}, \ldots, a_{c}$ in $R$ is exact if $a_{i}$ is exact on $R /\left(a_{1}, \ldots, a_{i-1}\right)$ for $i=1, \ldots, c$. An ideal that can be generated by an exact sequence is called exact.

Theorem 3.7. Let $a_{1}, \ldots, a_{c}$ be an exact sequence in $R$ and set $I=\left(a_{1}, \ldots, a_{c}\right)$.

The ideal I then is quasi-complete intersection, and for $S=R / I$ one has

$$
\operatorname{grade}_{R} S=\operatorname{card}\left\{i \in[1, c] \mid a_{i} \text { is regular on } R /\left(a_{1}, \ldots, a_{i-1}\right)\right\} \text {. }
$$

Proof. For $c=1$ see 3.1 , so fix $c \geq 2$. Set $I^{\prime}=\left(a_{1}, \ldots, a_{c-1}\right)$ and $R^{\prime}=R / I^{\prime}$. Let $E^{\prime}$ and $E$ be the Koszul complexes on $\left\{a_{1}, \ldots, a_{c-1}\right\}$ and $\left\{a_{1}, \ldots, a_{c}\right\}$, respectively. By induction, we assume $\mathrm{H}_{*}\left(E^{\prime}\right) \cong \Lambda_{*}^{R^{\prime}}\left(\mathrm{H}_{1}\left(E^{\prime}\right)\right)$ as algebras and $\mathrm{H}_{1}\left(E^{\prime}\right)$ is $R^{\prime}$-free.

Considering $E^{\prime}$ as a DG subalgebra of $E$, set $a=a_{c}$, and choose $e \in E_{1}$ with $\partial(e)=a$. Thus, the elements of $E$ have the form $y^{\prime}+e y^{\prime \prime}$ with unique $y^{\prime}, y^{\prime \prime} \in E^{\prime}$, and $E / E^{\prime} \cong \Sigma E^{\prime}$ as DG $E$-modules; see 2.8. The exact sequence of DG E-modules

$$
0 \rightarrow E^{\prime} \rightarrow E \rightarrow E / E^{\prime} \rightarrow 0
$$

induces in homology an exact sequence of $R^{\prime}$-modules

$$
\cdots \rightarrow \mathrm{H}_{n}\left(E^{\prime}\right) \stackrel{a}{\rightarrow} \mathrm{H}_{n}\left(E^{\prime}\right) \rightarrow \mathrm{H}_{n}(E) \rightarrow \mathrm{H}_{n-1}\left(E^{\prime}\right) \stackrel{a}{\rightarrow} \mathrm{H}_{n-1}\left(E^{\prime}\right) \rightarrow \cdots
$$

and hence an exact sequence of graded $\mathrm{H}_{*}\left(E^{\prime}\right)$-modules

$$
0 \rightarrow \mathrm{H}_{*}\left(E^{\prime}\right) / a \mathrm{H}_{*}\left(E^{\prime}\right) \rightarrow \mathrm{H}_{*}(E) \rightarrow \Sigma(0: a)_{\mathrm{H}_{*}\left(E^{\prime}\right)} \rightarrow 0
$$

Let $H^{\prime}$ be the image of $\mathrm{H}_{*}\left(E^{\prime}\right) / a \mathrm{H}_{*}\left(E^{\prime}\right)$ in $\mathrm{H}_{*}(E)$. It is a graded $S$-subalgebra, with $H^{\prime} \cong \mathrm{H}_{*}\left(E^{\prime}\right) / a \mathrm{H}_{*}\left(E^{\prime}\right)$ as graded algebras and $H_{1}^{\prime}$ free over $S$.

If $a$ is $R^{\prime}$-regular, then $(0: a)_{\mathrm{H}_{*}\left(E^{\prime}\right)}=0$, so $\mathrm{H}_{*}(E)=H^{\prime}$ by (3.7.1). This implies $\mathrm{H}_{*}(E) \cong \Lambda_{*}^{S}\left(\mathrm{H}_{1}(E)\right)$, and hence $\operatorname{grade}_{R} S=\operatorname{grade}_{R} R^{\prime}+1$ by (1.2.1).

If $a$ is an exact zero-divisor, we have $(0: a)_{\mathrm{H}_{*}\left(E^{\prime}\right)} \cong \mathrm{H}_{*}\left(E^{\prime}\right) / a \mathrm{H}_{*}\left(E^{\prime}\right)$ because $\mathrm{H}_{*}\left(E^{\prime}\right)$ is $R^{\prime}$-free. Thus, (3.7.1) gives $\mathrm{H}_{*}(E) \cong H^{\prime} \oplus \Sigma H^{\prime}$ as graded $H^{\prime}$-modules. It follows that there exists an element $h$ in $\mathrm{H}_{1}(E)$, such that $\mathrm{H}_{1}(E)=H_{1}^{\prime} \oplus R^{\prime} h$ 
and $h^{\prime} h=(-1)^{\left|h^{\prime}\right|} h h^{\prime}$ holds for $h^{\prime} \in H^{\prime}$. Since $h^{2}=0$ we get an isomorphism of algebras $\mathrm{H}_{*}(E) \cong \Lambda_{*}^{S}\left(\mathrm{H}_{1}(E)\right)$, and then $\operatorname{grade}_{R} S=\operatorname{grade}_{R} R^{\prime}$ follows from (1.2.1).

Remark 3.8. Theorem 8.8, proved by using André-Quillen homology, gives an independent proof that exact ideals are quasi-complete intersection. In an earlier version of this paper we had asked whether the converse holds. Since then, a negative answer has been obtained by Kustin, Şega, and Vraciu [31].

We finish with examples of exact zero-divisors, taken from the literature.

Example 3.9. Let $k$ be an algebraically closed field and $\underline{R}$ a graded ring of the form $k\left[x_{1}, \ldots, x_{e}\right] / \underline{I}$, with $\operatorname{deg}\left(x_{i}\right)=1$ and $\underline{I}$ generated by forms of degree at least 2 .

The quadratic $k$-algebras $\underline{R}$ with $\operatorname{rank}_{k} \underline{R}_{2}=e-1$ are parametrized by the points of the Grassmannian of subspaces of rank $\left(\begin{array}{c}e \\ 2\end{array}\right)+1$ in the $\left(\begin{array}{c}e+1 \\ 2\end{array}\right)$-dimensional affine space over $k$. Conca [22, Theorem 1] proved that this Grassmannian contains a non-empty open subset $U$, such that each $\underline{R}$ in $U$ contains an element $a \in \underline{R}_{1}$ with $a^{2}=0$ and $a \underline{R}_{1}=\underline{R}_{2} \neq 0$. This $\operatorname{implies}_{\operatorname{rank}_{k}}(a)=e$ and $(a \underline{R}) \subseteq(0: a)_{\underline{R}}$. A length count shows that equality holds, so $a$ is an exact zero-divisor.

Example 3.10. Let $k$ and $\underline{R}$ be as in the preceding example.

The Gorenstein $k$-algebras $\underline{R}$ with $\underline{R}_{4}=0 \neq \underline{R}_{3}$ are parametrized by the points of the $\left(\begin{array}{c}e+2 \\ 3\end{array}\right)$-dimensional projective space over $k$. This space contains a non-empty open subset $U$, such that each $\underline{R} \in U$ contains some exact zero-divisor $a \in \underline{R}_{1}$ : this follows from Conca, Rossi, and Valla [23, 2.13]; see also [29, 3.5].

\section{Grade}

In this section $(R, \mathfrak{m}, k)$ denotes a local ring, $I$ is an ideal of $R$, and $S=R / I$.

Recall that $(R, \mathfrak{m}, k)$ is said to be quasi-homogeneous if there is a finitely generated graded $k$-algebra $\underline{R}=\bigoplus_{i=0}^{\infty} \underline{R}_{i}$ with $\underline{R}_{0}=k$, such that the $\mathfrak{m}$-adiccompletion $\widehat{R}$ of $R$ is isomorphic to the $\left(\bigoplus_{i=1}^{\infty} \underline{R}_{i}\right)$-adic completion $\underline{\widehat{R}}$ of $\underline{R}$. The ideal $I$ is quasi-homogeneous if $I=\underline{I} \widehat{R}$ for some homogeneous ideal $\underline{I}$ of $\underline{R}$. 
Theorem 4.1. If I is quasi-complete intersection, then

$$
\operatorname{grade}_{R} S=\operatorname{depth} R-\operatorname{depth} S .
$$

Furthermore, an equality

$$
\operatorname{grade}_{R} S=\operatorname{dim} R-\operatorname{dim} S
$$

holds when one of the following conditions is satisfied:

(a) $\mathrm{CI}-\operatorname{dim}_{R} S$ is finite.

(b) $I$ is exact.

(c) I is quasi-homogeneous.

Proof. In view of Theorem 2.5(4), the equality (4.1.1) follows from (2.4.1).

For (4.1.2) we give a different argument in each case.

(a) Lemma 2.7 gives a local ring $Q$, complete intersection ideals $J \subseteq I^{\prime}$ of $Q$, and a flat local homomorphism $R \rightarrow Q / J$ with $I(Q / J)=I^{\prime} / J$. For $R^{\prime}=Q / J$ and $S^{\prime}=S \otimes_{R} R^{\prime}$ we have $\operatorname{dim} R-\operatorname{dim} S=\operatorname{dim} R^{\prime}-\operatorname{dim} S^{\prime}$ because $S \rightarrow S^{\prime}$ is a flat local homomorphism with $k \otimes_{S} S^{\prime} \cong k \otimes_{R} R^{\prime}$. As $J$ and $I^{\prime}$ are generated by regular sequences we have $\operatorname{dim} R^{\prime}-\operatorname{dim} S^{\prime}=\operatorname{grade}_{R^{\prime}} S^{\prime}$. Flatness yields for each $n$ an isomorphism $\operatorname{Ext}_{R^{\prime}}^{n}\left(S^{\prime}, R^{\prime}\right) \cong \operatorname{Ext}_{R}^{n}(S, R) \otimes_{R} R^{\prime}$, hence grade $R^{\prime} S^{\prime}=\operatorname{grade}_{R} S$.

(b) By hypothesis, $I=\left(a_{1}, \ldots, a_{c}\right)$ for some exact sequence $a_{1}, \ldots, a_{c}$. Set $d=$ $\operatorname{dim} R$ and let $a$ be an exact element of $R$. If $a$ is regular, then $\operatorname{dim} R /(a)=d-1$, so in view of Theorem 3.7 it suffices to show that $\operatorname{dim} R /(a)=d$ holds when $a$ is an exact zero-divisor. This is evident for $d=0$, so we further assume $d \geq 1$.

Suppose, by way of contradiction, that $\operatorname{dim} R /(a)<d$ holds. Choose $\mathfrak{q}$ in $\operatorname{Spec} R$ with $\operatorname{dim}(R / \mathfrak{q})=d$. We must have $a \notin \mathfrak{q}$, whence

$$
\operatorname{dim} R /(a) \leq d-1=\operatorname{dim} R /(\mathfrak{q}+(a)) \leq \operatorname{dim} R /(a)
$$

Thus, some prime ideal $\mathfrak{p}$ containing $\mathfrak{q}+(a)$ satisfies $\operatorname{dim}(R / \mathfrak{p})=\operatorname{dim} R /(a)$. It follows that $\mathfrak{p}$ is minimal over $(a)$. Krull's Principal Ideal Theorem now gives height $\mathfrak{p} \leq 1$; in fact, equality holds, due to the inclusion $\mathfrak{p} \supsetneq \mathfrak{q}$.

Now choose a generator $b$ of $(0: a)_{R}$. The exact sequence of $R$-modules

$$
0 \rightarrow R /(a) \rightarrow R \rightarrow R /(b) \rightarrow 0
$$


yields $(R /(b))_{\mathfrak{q}} \cong R_{\mathfrak{q}} \neq 0$, hence $b \in \mathfrak{q} \subseteq \mathfrak{p}$. Localizing at $\mathfrak{p}$ and changing notation, we get a local $\operatorname{ring}(R, \mathfrak{m}, k)$ with $\operatorname{dim} R=1$ and elements $a$ and $b$ in $\mathfrak{m}$, such that the map $R /(a) \rightarrow R$ given by $c+(a) \mapsto b c$ is injective. A result of Fouli and Huneke, $[25,4.1]$, implies that $a b$ is a parameter for $R$; this is absurd, as $a b=0$.

(c) The ingredients of formula (4.1.2) do not change under m-adic completion, so we may assume $R=\underline{\widehat{R}}$ and $I=\underline{I} R$, with $\underline{I}$ minimally generated by homogeneous elements $\underline{a}_{1}, \ldots, \underline{a}_{c}$ in $\underline{R}$. The Koszul complex $\underline{E}$ on these generators is naturally bigraded, so there exist homogeneous cycles $\underline{z}_{1}, \ldots, \underline{z}_{h}$ in $\underline{E}_{1}$, whose homology classes minimally generate the graded $\underline{R}$-module $\mathrm{H}_{1}(\underline{E})$. Construction 1.5 then yields a complex $\underline{F}$ of graded $\underline{R}$-modules, with differentials of degree 0 .

The complex of $R$-modules $R \otimes_{\underline{R}} \underline{F}$ is isomorphic to the Tate complex $F$ on $R \otimes_{\underline{R}} \underline{E}$ and $\left\{1 \otimes \underline{z}_{1}, \ldots, 1 \otimes \underline{z}_{h}\right\}$, which is a free resolution of $S$ over $R$ by 1.6. On the category of finite graded $\underline{R}$-modules the functor $\left(R \otimes_{\underline{R}}-\right)$ is exact and faithful, so $\underline{F}$ is a graded free resolution of $\underline{S}=\underline{R} / \underline{I}$ over $\underline{R}$. As $\left(\underline{F}_{n}\right)_{j}=0$ for $j<n$, we get

$$
\sum_{n=0}^{\infty}\left(\sum_{j=n}^{\infty} \operatorname{rank}_{\underline{R}}\left(\underline{F}_{n}\right)_{j} s^{j}\right) t^{n}=\frac{\prod_{u=1}^{c}\left(1+s^{\operatorname{deg} a_{u}} t\right)}{\prod_{v=1}^{h}\left(1-s^{\operatorname{deg} z_{v}} t^{2}\right)} .
$$

by counting ranks of graded free $\underline{R}$-modules. Set $H_{S}(s)=\sum_{j \in \mathbb{Z}} \operatorname{rank}_{k} S_{j} s^{n} \in$ $\mathbb{Z}[[s]]$ and define $H_{R}(s)$ similarly. By counting $k$-ranks in the exact sequence

$$
\cdots \rightarrow \underline{F}_{n} \rightarrow \underline{F}_{n-1} \rightarrow \cdots \rightarrow \underline{F}_{1} \rightarrow \underline{F}_{0} \rightarrow \underline{S} \rightarrow 0
$$

we obtain the first equality of formal power series in the following formula:

$$
H_{\underline{S}}(s)=H_{\underline{R}}(s) \cdot \sum_{n=0}^{\infty}(-1)^{n}\left(\sum_{j=n}^{\infty} \operatorname{rank}_{\underline{R}}\left(\underline{F}_{n}\right)_{j} s^{j}\right)=H_{\underline{R}}(s) \cdot \frac{\prod_{u=1}^{c}\left(1-s^{\operatorname{deg} a_{u}}\right)}{\prod_{v=1}^{h}\left(1-s^{\operatorname{deg} z_{v}}\right)} .
$$

The second equality is obtained by evaluating formula (4.1.3) at $t=-1$.

By the Hilbert-Serre Theorem, $H_{\underline{S}}(s)$ represents a rational function in $s$, and the order of its pole at $s=1$ equals the Krull dimension of $\underline{S}$. Equating the orders of the poles at $s=1$ in the formula above, we get the second equality in the string

$$
\operatorname{dim} S=\operatorname{dim} \underline{S}=\operatorname{dim} \underline{R}+h-c=\operatorname{dim} R+h-c=\operatorname{dim} R-\operatorname{grade}_{R} S .
$$

The last equality is given by (1.2.1), while the other two are standard.

The theorem above supports the following 
Conjecture 4.2. The equality (4.1.2) holds for each quasi-complete intersection ideal.

Remark 4.3. It suffices to prove Conjecture 4.2 in the special case $\operatorname{grade}_{R} S=0$.

Indeed, set $d=\operatorname{dim} R$ and $g=\operatorname{grade}_{R} S$. By prime avoidance, $I$ has a minimal generating set in which the first $g$ elements form an $R$-regular sequence, $\boldsymbol{b}$. By Lemma 1.4, the ideal $\bar{I}=I /(\boldsymbol{b})$ of $\bar{R}=R /(\boldsymbol{b})$ is quasi-complete intersection. We now have $S \cong \bar{R} / \bar{I}$ with $\operatorname{grade}_{\bar{R}} S=0$, and $\operatorname{dim} \bar{R}=d-g$.

Recall that a finite $R$-module $M$ is called totally reflexive if $M \cong M^{\vee \vee}$ and $\operatorname{Ext}_{R}^{n}(M, R)=0=\operatorname{Ext}_{R}^{n}\left(M^{\vee}, R\right)$ for $n \geq 1$; here $(-)^{\vee}$ stands for $\operatorname{Hom}_{R}(-, R)$.

Remark 4.4. A quasi-complete intersection ideal $I$ is quasi-Gorenstein by Theorem 2.5(4), so when $\operatorname{grade}_{R} S=0$ the $R$-module $S$ is totally reflexive by 2.4 . Remark 4.3 now shows that a positive answer to the next question implies Conjecture 4.2 .

Question 4.5. Does $\operatorname{dim}_{R} M=\operatorname{dim} R$ hold for every totally reflexive $R$-module $M ?$

Remark 4.6. When $R$ is Cohen-Macaulay the answer to Question 4.5 is positive: If $M$ is totally reflexive, then for every $n \geq 0$ it is an $n$th syzygy module in some minimal free resolution, so $\operatorname{dim}_{R} M=\operatorname{depth} R$; see, for instance, [7, 1.2.8].

\section{Homology ALGEBRAS}

When $(R, \mathfrak{m}, k)$ is a local ring $\operatorname{Tor}_{*}^{R}(k, k)$ has a structure graded-commutative $k$-algebra. It is natural in $R$, so each ideal $I$ defines a homomorphism of graded $k$-algebras $\operatorname{Tor}_{*}^{R}(k, k) \rightarrow \operatorname{Tor}_{*}^{R / I}(k, k)$. One of the few cases where it is fully understood is when $I$ is generated by a regular element; see [28, 3.4.1].

Without using that information, in this section we obtain an explicit result for all quasi-complete intersection ideals. It plays an important role when analyzing the transfer of properties between $R$ and $S$; see Proposition 7.5.

5.1. A system of divided powers on a graded $R$-algebra $A$ is an operation that for each $j \geq 1$ and each $i \geq 0$ assigns to every $a \in A_{2 i}$ an element $a^{(j)} \in A_{2 i j}$, subject to certain axioms; cf. $[28,1.7 .1]$. A DG $R$-algebra is a DG $R$-algebra $A$ with divided powers compatible with the differential: $\partial\left(a^{(j)}\right)=\partial(a) a^{(j-1)}$. 
Let $A$ be a DG $\Gamma$ algebra with $R \rightarrow A_{0}$ surjective and $A_{n}=0$ for $n<0$. Let $A_{\geqslant 1}$ denote the subset of $A$, consisting of elements of positive degree. Let $D(A)$ denote the graded $R$-submodule of $A$, generated by $A_{0}+\mathfrak{m} A_{\geqslant 1}$, all elements of the form $u v$ with $u, v \in A_{\geqslant 1}$, and all $w^{(j)}$ with $w \in A_{2 i}, i \geq 1, j \geq 2$. This clearly is a subcomplex of $A$, and it defines a complex $Q^{\gamma}(A)=A / D(A)$ of $k$-vector spaces.

Given a set $\boldsymbol{x}$ of variables with $|x| \geq 1$ for all $x \in \boldsymbol{x}$ (where $|x|$ denotes the degree of $x$ ), we let $A\langle\boldsymbol{x}\rangle$ denote a DG $\mathrm{D}$ algebra with

$$
A \otimes_{R} \Lambda_{*}^{R}\left(\bigoplus_{\substack{x \in \boldsymbol{x} \\|x| \text { odd }}} R x\right) \otimes_{R} \Gamma_{*}^{R}\left(\bigoplus_{\substack{x \in \boldsymbol{x} \\|x| \text { even }}} R x\right)
$$

as underlying graded algebra and differential compatible with that of $A$ and the divided powers of $x \in \boldsymbol{x}$. For every integer $n$ we set $\boldsymbol{x}_{n}=\{x \in \boldsymbol{x}:|x|=n\}$.

A Tate resolution of a surjective ring homomorphism $R \rightarrow T$ is a quasi-isomorphism $R\langle\boldsymbol{x}\rangle \rightarrow T$, where $\boldsymbol{x}=\left\{x_{i}\right\}_{i \geqslant 1}$ and $\left|x_{j}\right| \geq\left|x_{i}\right| \geq 1$ holds for all $j \geq i \geq 1$. Such a resolution always exists: see [41, Thm. 1], [28, 1.2.4], or [7, 6.1.4].

5.2. Any Tate resolution of $R \rightarrow k$ gives $\operatorname{Tor}_{*}^{R}(k, k)$ a structure of DG $k$-algebra, and this structure is independent of the choice of resolution. We set

$$
\pi_{*}(R)=Q^{\gamma}\left(\operatorname{Tor}_{*}^{R}(k, k)\right)
$$

We use the following natural isomorphisms as identifications:

$$
\pi_{1}(R) \cong \operatorname{Tor}_{1}^{R}(k, k) \cong \mathfrak{m} / \mathfrak{m}^{2}
$$

The assignment $R \mapsto \pi_{*}(R)$ is a functor from the category of local rings and surjective homomorphisms to that of graded $k$-vector spaces.

Theorem 5.3. Let $(R, \mathfrak{m}, k)$ be a local ring and $I$ an ideal of $R$. Set $S=R / I$ and let $\varphi: R \rightarrow S$ be the natural map. Let $E$ be the Koszul complex on a minimal generating set of $I$ and set $H=\mathrm{H}_{1}(E)$.

If $I$ is quasi-complete intersection, then there is an exact sequence

$$
0 \rightarrow H / \mathfrak{m} H \rightarrow \pi_{2}(R) \stackrel{\pi_{2}(\varphi)}{\longrightarrow} \pi_{2}(S) \stackrel{\delta}{\rightarrow} I / \mathfrak{m} I \rightarrow \pi_{1}(R) \stackrel{\pi_{1}(\varphi)}{\longrightarrow} \pi_{1}(S) \rightarrow 0
$$

of $k$-vector spaces, and there are isomorphisms of $k$-vector spaces

$$
\pi_{n}(\varphi): \pi_{n}(R) \stackrel{\cong}{\longrightarrow} \pi_{n}(S) \text { for } n \geq 3
$$


Remark. The statement of the theorem and its proof are reminiscent of those of $[5,1.1]$, but neither result implies the other one.

Parts of the theorem can also be obtained by using André-Quillen homology.

Indeed, $I / \mathfrak{m} I \cong \mathrm{D}_{1}(S \mid R, k)$ holds by $[1,6.1], H / \mathfrak{m} H \cong \mathrm{D}_{2}(S \mid R, k)$ by $[1,15.12]$, and $\pi_{n}(R) \cong \mathrm{D}_{n}(k \mid R, k)$ for $n=1,2$ by [1, 6.1 and 15.8]. Furthermore, (A.1.1) applied to the homomorphisms $R \rightarrow S \rightarrow k \stackrel{\equiv}{\rightarrow}$ yields an exact sequence

$$
\cdots \rightarrow \mathrm{D}_{n}(k \mid R, k) \longrightarrow \mathrm{D}_{n}(k \mid S, k) \longrightarrow \mathrm{D}_{n}(S \mid R, k) \stackrel{\partial_{n}}{\longrightarrow} \mathrm{D}_{n-1}(k \mid R, k) \rightarrow \cdots
$$

If $\mathrm{H}_{1}(E)$ is free, then $\partial_{3}$ is injective by [35, Theorem 1]; this yields (5.3.1).

For $n \geq 3$ one has $\mathrm{D}_{n}(S \mid R, k)=0$ by 1.7 . If $\operatorname{char}(k)=0$, then $[1,19.21]$ gives $\pi_{n}(R) \cong \mathrm{D}_{n}(k \mid R, k)$ for all $n$, so in this case (5.3.2) follows from (5.3.3). However, $\pi_{*}(R)$ and $\mathrm{D}_{*}(k \mid R, k)$ are not isomorphic when $\operatorname{char}(k)>0$; see [2].

Construction 5.4. Choose a Tate resolution $S\langle\boldsymbol{y}\rangle \rightarrow k$ with $\partial(S\langle\boldsymbol{y}\rangle) \subseteq \mathfrak{m}(S\langle\boldsymbol{y}\rangle)$ : this is always possible, see $[28,1.6 .4]$ or $[7,6.3 .5]$.

In view of 1.6, the complex $F$ from Construction 1.5 gives a Tate resolution $R\langle\boldsymbol{v}, \boldsymbol{w}\rangle \rightarrow S$. This map can be extended to a surjective quasi-isomorphism

$$
\alpha: R\langle\boldsymbol{v}, \boldsymbol{w}, \boldsymbol{x}\rangle \rightarrow S\langle\boldsymbol{y}\rangle
$$

of DGC algebras with $\boldsymbol{x}=\left\{x_{i}\right\}_{i \geqslant 1}$ and $\alpha\left(x_{i}\right)=y_{i}$ for each $i$; see [28, 1.3.5]. In particular, $R\langle\boldsymbol{v}, \boldsymbol{w}, \boldsymbol{x}\rangle$ is a free, not necessarily minimal, resolution of $k$ over $R$.

Proof of Theorem 5.3. The homomorphisms of DGT algebras

$$
R\langle\boldsymbol{v}, \boldsymbol{w}\rangle \hookrightarrow R\langle\boldsymbol{v}, \boldsymbol{w}, \boldsymbol{x}\rangle \stackrel{\alpha}{\rightarrow} S\langle\boldsymbol{y}\rangle
$$

induce an exact sequence of complexes of $k$-vector-spaces:

$$
0 \rightarrow Q^{\gamma}(R\langle\boldsymbol{v}, \boldsymbol{w}\rangle) \rightarrow Q^{\gamma}(R\langle\boldsymbol{v}, \boldsymbol{w}, \boldsymbol{x}\rangle) \stackrel{Q^{\gamma}(\alpha)}{\longrightarrow} Q^{\gamma}(S\langle\boldsymbol{y}\rangle) \rightarrow 0
$$

Due to the inclusions $\partial(R\langle\boldsymbol{v}, \boldsymbol{w}\rangle) \subseteq \mathfrak{m} R\langle\boldsymbol{v}, \boldsymbol{w}\rangle$ and $\partial(S\langle\boldsymbol{y}\rangle) \subseteq \mathfrak{m} S\langle\boldsymbol{y}\rangle$, the complexes at both ends of (5.5.1) have zero differentials. This gives the following 
expressions:

$$
\begin{aligned}
\mathrm{H}_{n}\left(Q^{\gamma}(R\langle\boldsymbol{v}, \boldsymbol{w}\rangle)\right. & = \begin{cases}k \boldsymbol{v} \cong I / \mathfrak{m} I & \text { for } n=1, \\
k \boldsymbol{w} \cong H / \mathfrak{m} H & \text { for } n=2, \\
0 & \text { for } n \neq 1,2 .\end{cases} \\
\mathrm{H}_{n}\left(Q^{\gamma}(S\langle\boldsymbol{y}\rangle)=k \boldsymbol{y}_{n} \cong \pi_{n}(S)\right. & \text { for all } n
\end{aligned}
$$

The next statement is the key point of the argument.

Claim. For $G=R\langle\boldsymbol{v}, \boldsymbol{w}, \boldsymbol{x}\rangle$ one has $\partial_{n}(G) \subseteq \mathfrak{m} D_{n-1}(G)$ for $n \neq 2$.

Indeed, for $n \neq 3$ this follows from the exact sequence (5.5.1) and the equalities in (5.5.2) and (5.5.3). Next we prove $\partial\left(G_{3}\right) \subseteq \mathfrak{m} G_{2}+D_{2}(G)$. Write $z \in \partial\left(G_{3}\right)$ as

$$
z=\sum_{x \in \boldsymbol{x}_{2}} a_{x} x+\sum_{w \in \boldsymbol{w}} b_{w} w+c .
$$

with $a_{x}$ and $b_{w}$ in $R$ and $c \in D_{2}(G)$. One has $\partial(\alpha(z))=\alpha(\partial(z))=0$, so $\alpha(z)$ is a cycle in the minimal free resolution $S\langle\boldsymbol{y}\rangle$; this gives the inclusion below:

$$
\sum_{x \in \boldsymbol{x}_{2}} \varphi\left(a_{x}\right) y+\alpha(c)=\alpha(z) \in \mathfrak{m} S\langle\boldsymbol{y}\rangle
$$

From this formula, we conclude that in (5.5.4) we have $a_{x} \in \mathfrak{m}$ for each $x \in \boldsymbol{x}_{2}$.

Now we show that in (5.5.4) each $b_{w}$ is in $\mathfrak{m}$. Assume, by way of contradiction, that $b_{w} \notin \mathfrak{m}$ holds for some $w \in \boldsymbol{w}$. Note that $R\left\langle\boldsymbol{v}, \boldsymbol{w}, \boldsymbol{x}_{1}, \boldsymbol{x}_{2}\right\rangle$ has an $R$-basis consisting of products involving elements from $\boldsymbol{v} \sqcup \boldsymbol{x}_{1}$ and divided powers of elements from $\boldsymbol{w} \sqcup \boldsymbol{x}_{2}$. When the boundary of an element of $R\left\langle\boldsymbol{v}, \boldsymbol{w}, \boldsymbol{x}_{1}, \boldsymbol{x}_{2}\right\rangle$ is written in this basis, the coefficient of $w^{(j)}$ cannot be invertible; this follows from the Leibniz rule. On the other hand, the defining properties of divided powers, see $[28,1.7 .1]$, imply that in the expansion of $z^{(j)}$ the element $w^{(j)}$ appears with coefficient $\left(b_{w}\right)^{j}$. In homology, this means $\operatorname{cls}(z)^{(j)}=\operatorname{cls}\left(z^{(j)}\right) \neq 0$. Since $\alpha$ is a quasi-isomorphism, for every $j \geq 1$ we get $\operatorname{cls}(\alpha(z))^{(j)} \neq 0$ in $\mathrm{H}_{*}\left(S\left\langle\boldsymbol{y}_{1}, \boldsymbol{y}_{2}\right\rangle\right)$. This is impossible, as for $j>\operatorname{rank}_{k}\left(\mathfrak{m} / \mathfrak{m}^{2}\right)$ the $j$ th divided power of every class of even degree in $\mathrm{H}_{*}\left(S\left\langle\boldsymbol{y}_{1}, \boldsymbol{y}_{2}\right\rangle\right)$ is equal to zero; see $[5,1.7]$. This finishes the proof of the claim.

The claim gives, in particular, $\partial_{n}\left(Q^{\gamma}(R\langle\boldsymbol{v}, \boldsymbol{w}, \boldsymbol{x}\rangle)\right)=0$ for all odd $n$. By [28, 3.2.1(iii) and its proof], there is a quasi-isomorphism $\rho: R\langle\boldsymbol{v}, \boldsymbol{w}, \boldsymbol{x}\rangle \rightarrow R\left\langle\boldsymbol{x}^{\prime}\right\rangle$ of DG $R$-algebras, such that $R\left\langle\boldsymbol{x}^{\prime}\right\rangle$ is a minimal DG $\Gamma$ algebra resolution of $k$, and 
the induced map $Q^{\gamma}(\rho): Q^{\gamma}(R\langle\boldsymbol{v}, \boldsymbol{w}, \boldsymbol{x}\rangle) \rightarrow Q^{\gamma}\left(R\left\langle\boldsymbol{x}^{\prime}\right\rangle\right)$ is a quasi-isomorphism. Choose, by [28, 1.8.6], a quasi-isomorphism $\sigma: R\left\langle\boldsymbol{x}^{\prime}\right\rangle \rightarrow R\langle\boldsymbol{v}, \boldsymbol{w}, \boldsymbol{x}\rangle$ of DG $R$ algebras. The minimality of $R\left\langle\boldsymbol{x}^{\prime}\right\rangle$ implies that the composition $\rho \sigma: R\left\langle\boldsymbol{x}^{\prime}\right\rangle \rightarrow$ $R\left\langle\boldsymbol{x}^{\prime}\right\rangle$ is an isomorphism, see [28, 1.9.5], hence so is the map $Q^{\gamma}(\rho \sigma)$. It is equal to $Q^{\gamma}(\rho) Q^{\gamma}(\sigma)$, so $Q^{\gamma}(\sigma)$ is a quasi-isomorphism. Now form the composition of $k$-linear maps

$$
\pi_{n}(R) \cong \mathrm{H}_{n}\left(Q^{\gamma}\left(R\left\langle\boldsymbol{x}^{\prime}\right\rangle\right)\right) \cong \mathrm{H}_{n}\left(Q^{\gamma}(R\langle\boldsymbol{v}, \boldsymbol{w}, \boldsymbol{x}\rangle)\right) \stackrel{\mathrm{H}_{n}\left(Q^{\gamma}(\alpha)\right)}{\longrightarrow} \mathrm{H}_{n}\left(Q^{\gamma}(S\langle\boldsymbol{y}\rangle)\right) \cong \pi_{n}(S)
$$

where the first isomorphism is due to the minimality of $R\left\langle\boldsymbol{x}^{\prime}\right\rangle$, the second one is $\mathrm{H}_{n}\left(Q^{\gamma}(\sigma)\right)$, and the third one is (5.5.3). As $\alpha \sigma: R\left\langle\boldsymbol{x}^{\prime}\right\rangle \rightarrow S\langle\boldsymbol{y}\rangle$ induces the identity on $k$, the composed map is, by definition, $\pi_{n}(\varphi): \pi_{n}(R) \rightarrow \pi_{n}(S)$. It follows that the homology exact sequence of the exact sequence (5.5.1) is isomorphic to

$$
\cdots \rightarrow \mathrm{H}_{n}\left(Q ^ { \gamma } ( R \langle \boldsymbol { v } , \boldsymbol { w } \rangle ) \rightarrow \pi _ { n } ( R ) \stackrel { \pi _ { n } ( \varphi ) } { \longrightarrow } \pi _ { n } ( S ) \stackrel { \partial _ { n } } { \longrightarrow } \mathrm { H } _ { n - 1 } \left(Q^{\gamma}(R\langle\boldsymbol{v}, \boldsymbol{w}\rangle) \rightarrow \cdots\right.\right.
$$

In view of the isomorphisms in (5.5.2), it remains to prove $\partial_{3}=0$. This follows from the construction of the connecting isomorphism, and the claim for $n=3$.

\section{Poincaré Series}

In this section $(R, \mathfrak{m}, k)$ is a local ring, $I$ an ideal, $S=R / I$, and $N$ a finite $S$-module. Recall that the Poincaré series of $N$ over $S$ is the formal power series

$$
P_{N}^{S}(t)=\sum_{n=0}^{\infty} \operatorname{rank}_{k} \operatorname{Tor}_{n}^{S}(k, N) t^{n} .
$$

Our goal is to relate it to $P_{N}^{R}(t)$ when $I$ is quasi-complete intersection. The case $N=k$ is of special interest, as the Poincaré series of the residue field encodes important information on how far the ring is from being regular.

The deviations of $S$ are defined using the vector spaces in (5.2.1), by the formula

$$
\varepsilon_{n}(S)=\operatorname{rank}_{k} \pi_{n}(S) \text { for } n \in \mathbb{Z} .
$$

They appear in a well-known formula, see $[28,3.1 .3]$ or $[7,7.1 .3]$ :

$$
P_{k}^{S}(t)=\frac{\prod_{i=0}^{\infty}\left(1+t^{2 i+1}\right)^{\varepsilon_{2 i+1}(S)}}{\prod_{i=0}^{\infty}\left(1-t^{2 i+2}\right)^{\varepsilon_{2 i+2}(S)}} .
$$

For the next theorems, recall that edim $R$ stands for $\operatorname{rank}_{k}\left(\mathfrak{m} / \mathfrak{m}^{2}\right)$. 
Theorem 6.1. When I is quasi-complete intersection the following equality hold$s$ :

$$
P_{k}^{S}(t) \cdot \frac{(1-t)^{\operatorname{edim} S}}{\left(1-t^{2}\right)^{\operatorname{depth} S}}=P_{k}^{R}(t) \cdot \frac{(1-t)^{\operatorname{edim} R}}{\left(1-t^{2}\right)^{\operatorname{depth} R}} .
$$

Proof. Set $g=\operatorname{grade}_{R} S, h=\operatorname{rank}_{S} \mathrm{H}_{1}(E)$, and $c=\operatorname{rank}_{k} I / \mathfrak{m} I$. The equalities

$$
\begin{aligned}
P_{k}^{S}(t) & =\frac{(1+t)^{\varepsilon_{1}(S)}}{\left(1-t^{2}\right)^{c-h+\varepsilon_{1}(S)-\varepsilon_{1}(R)+\varepsilon_{2}(R)}} \cdot \frac{\prod_{i=1}^{\infty}\left(1+t^{2 i+1}\right)^{\varepsilon_{2 i+1}(S)}}{\prod_{i=1}^{\infty}\left(1-t^{2 i+2}\right)^{\varepsilon_{2 i+2}(S)}} \\
& =\frac{1}{\left(1-t^{2}\right)^{g}} \cdot \frac{(1-t)^{\varepsilon_{1}(R)}}{(1-t)^{\varepsilon_{1}(S)}} \cdot \frac{(1+t)^{\varepsilon_{1}(R)}}{\left(1-t^{2}\right)^{\varepsilon_{2}(R)}} \cdot \frac{\prod_{i=1}^{\infty}\left(1+t^{2 i+1}\right)^{\varepsilon_{2 i+1}(R)}}{\prod_{i=1}^{\infty}\left(1-t^{2 i+2}\right)^{\varepsilon_{2 i+2}(R)}} \\
& =\frac{\left(1-t^{2}\right)^{\operatorname{depth} S}}{\left(1-t^{2}\right)^{\operatorname{depth} R}} \cdot \frac{(1-t)^{\varepsilon_{1}(R)}}{(1-t)^{\varepsilon_{1}(S)}} \cdot P_{k}^{R}(t)
\end{aligned}
$$

are obtained by applying (6.0.2) and (5.3.1) for the first one, (1.2.1) and (5.3.2) for the second, (4.1.1) and (6.0.2) for the third. Finally, $\varepsilon_{1}(S)=\operatorname{edim} S$ by (5.2.2).

Theorem 6.2. If $I$ is a quasi-complete intersection ideal satisfying $I \cap \mathfrak{m}^{2} \subseteq I \mathfrak{m}$, then for every finite $S$-module $N$ the following equality holds:

$$
P_{N}^{S}(t) \cdot \frac{(1-t)^{\operatorname{edim} S}}{\left(1-t^{2}\right)^{\operatorname{depth} S}}=P_{N}^{R}(t) \cdot \frac{(1-t)^{\operatorname{edim} R}}{\left(1-t^{2}\right)^{\operatorname{depth} R}} .
$$

Proof. As $I$ is quasi-complete intersection, the map $\pi_{n}(\varphi): \pi_{n}(R) \rightarrow \pi_{n}(S)$ is surjective for $n \neq 2$, by Theorem 5.3. The hypothesis $I \cap \mathfrak{m}^{2} \subseteq \mathfrak{m} I$ implies that $I / \mathfrak{m} I \rightarrow \mathfrak{m} / \mathfrak{m}^{2}$ is injective, so the same theorem shows that $\pi_{2}(\varphi)$ is surjective as well. By the definition of $\pi_{*}(R)$, the image of any $k$-linear right inverse $\sigma: \pi_{*}(R) \rightarrow \operatorname{Tor}_{*}^{R}(k, k)$ of the natural surjection $\operatorname{Tor}_{*}^{R}(k, k) \rightarrow \pi_{*}(R)$ generates $\operatorname{Tor}_{*}^{R}(k, k)$ as a graded $\Gamma$-algebra over $k$. Thus, the surjectivity of $\pi_{*}(\varphi)$ mean$\mathrm{s}$ that the map of $\Gamma$-algebras $\operatorname{Tor}_{*}^{\varphi}(k, k): \operatorname{Tor}_{*}^{R}(k, k) \rightarrow \operatorname{Tor}_{*}^{S}(k, k)$ is surjective; that is, $\varphi$ is a large homomorphism. A theorem of Levin, [32, 1.1], then gives

$$
P_{N}^{S}(t) P_{k}^{R}(t)=P_{N}^{R}(t) P_{k}^{S}(t) .
$$

Now replace $P_{k}^{S}(t)$ with its expression from Theorem 6.1, and simplify.

To finish, we compare our results with earlier ones for complete intersections. 
Remark 6.3. Let $\boldsymbol{a}$ be a regular sequence and $I=(\boldsymbol{a})$.

Formula (6.1.1) then specializes to theorems of Tate [41, Theorem 4] and Scheja [37, Satz 1], and (6.2.1) to one of Nagata [33, 27.3]. It suffices to prove those theorems for principal ideals, but such a reduction is impossible here; see Remark 3.8.

When $\boldsymbol{a}$ lies in $\mathfrak{m} \operatorname{Ann}_{R} N$ one has edim $S=\operatorname{edim} R$, so (6.2.1) holds by a result of Shamash, [38, $\S 3$, Corollary (2)]; see also [7, 3.3.5(2)]. We know of no analog of that result for quasi-complete intersection ideals.

In Theorem 6.2 the hypothesis $I \cap \mathfrak{m}^{2} \subseteq I \mathfrak{m}$ is essential:

Example 6.4. For $R=k \llbracket x \rrbracket, S=R /\left(x^{2}\right)$, and $N=S$ one has

$$
P_{N}^{S}(t) \cdot \frac{(1-t)^{\operatorname{edim} S}}{\left(1-t^{2}\right)^{\operatorname{depth} S}}=1-t \neq 1=P_{N}^{R}(t) \cdot \frac{(1-t)^{\operatorname{edim} R}}{\left(1-t^{2}\right)^{\operatorname{depth} R}} .
$$

\section{LOCAL HOMOMORPHISMS OF LOCAL RINGS}

In this section $\varphi:(R, \mathfrak{m}, k) \rightarrow(S, \mathfrak{n}, l)$ denotes a homomorphism of local rings, which is local, in the sense that it satisfies $\varphi(\mathfrak{m}) \subseteq \mathfrak{n}$.

We define and study quasi-complete intersection local homomorphisms, with an emphasis on the transfer of local ring-theoretic properties between $R$ and $S$.

By $[13,1.1]$, there is a commutative diagram of local homomorphisms



where $\dot{\varphi}$ is flat, $\varphi^{\prime}$ is surjective, $\sigma$ is the $\mathfrak{n} S$-adic completion map, $R^{\prime}$ is complete, and $R^{\prime} / \mathfrak{m} R^{\prime}$ is regular. Any such a diagram is called a Cohen factorization of $\varphi$.

7.1. We say that $\varphi: R \rightarrow S$ is quasi-complete intersection (or q.c.i.) at $\mathfrak{n}$ if in some Cohen factorization of $\varphi$ the ideal $\operatorname{Ker}\left(\varphi^{\prime}\right)$ is quasi-complete intersection. We first show that this property does not depend on the choice of Cohen factorization:

Lemma 7.2. The homomorphism $\varphi$ is q.c.i. at $\mathfrak{n}$ (if and) only if $\operatorname{Ker} \varphi^{\prime \prime}$ is quasicomplete intersection for every Cohen factorization $R \stackrel{\ddot{\varphi}}{\rightarrow} R^{\prime \prime} \stackrel{\varphi^{\prime \prime}}{\rightarrow} \widehat{S}$ of $\varphi$. 
Proof. By $[13,1.2]$, there is a commutative diagram of local homomorphisms

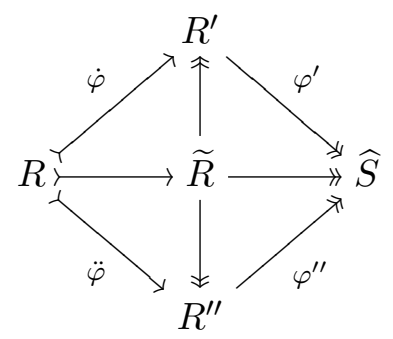

where the middle row is a Cohen factorization of $\varphi$, and the vertical arrows are surjections with kernels generated by regular sequences. Lemma 1.4 shows that $\operatorname{Ker}\left(\varphi^{\prime \prime}\right)$ is quasi-complete intersection if and only if $\operatorname{Ker}\left(\varphi^{\prime}\right)$ is.

Remark 7.3. When $\varphi$ is surjective, it is q.c.i. at $\mathfrak{n}$ if and only if the ideal $I=$ $\operatorname{Ker}(\varphi)$ is quasi-complete intersection.

Indeed, $R \rightarrow \widehat{R} \stackrel{\varphi}{\rightarrow} \widehat{S}$ clearly is a Cohen factorization, so by the preceding lemma it suffices to show that $I$ is quasi-complete intersection if and only if $\operatorname{Ker}(\widehat{\varphi})$ is one. In view of the equality $\operatorname{Ker}(\widehat{\varphi})=I \widehat{R}$, this follows from Lemma 1.3 .

The homomorphism $\varphi$ is said to be complete intersection (or c.i.), respectively, quasi-Gorenstein at $\mathfrak{n}$ if in some Cohen factorization the ideal $\operatorname{Ker}\left(\varphi^{\prime}\right)$ has the property described in 2.1, respectively, in 2.4. As above, this notion does not depend on the choice of factorization; see $[8,(3.3)]$ and $[11,4.3]$, respectively.

Proposition 7.4. The homomorphism $\varphi$ is c.i. at $\mathfrak{n}$ if and only if it is q.c.i. at $\mathfrak{n}$ and $S$ has finite flat dimension over $R$.

If $\varphi$ is q.c.i. at $\mathfrak{n}$, then it is quasi-Gorenstein at $\mathfrak{n}$.

Proof. Choose any Cohen factorization (7.0.1) and set $I=\operatorname{Ker}\left(\varphi^{\prime}\right)$.

By [13,3.3], $S$ has finite flat dimension over $R$ if and only if $I$ has finite projective dimension over $R^{\prime}$, so the first assertion follows from Theorem 2.5(1).

If $\varphi$ is q.c.i. at $\mathfrak{n}$, then the ideal $I$ is quasi-complete intersection by Lemma 7.2, and hence it is quasi-Gorenstein by Theorem 2.5(4).

Next we relate certain local properties of $R$ and $S$. If $\varphi$ is quasi-Gorenstein at $\mathfrak{n}$, then the rings $R$ and $S$ are simultaneously Gorenstein by [11, 7.7.2]. In view 
of 8.2 , the conclusion holds when $\varphi$ is q.c.i. at $\mathfrak{n}$; for surjective $\varphi$ this is already noted in [26, Cor.5], along with the fact that $S$ is Cohen-Macaulay when $R$ is.

We want to compare numerical measures of the singularity of local rings.

The Cohen-Macaulay defect of $S$ is the non-negative number

$$
\operatorname{cmd} S=\operatorname{dim} S-\operatorname{depth} S \text {; }
$$

it is equal to zero if and only if $S$ is Cohen-Macaulay.

Similarly, the complete intersection defect of $S$ is the non-negative number

$$
\operatorname{cid} S=\varepsilon_{2}(S)-\varepsilon_{1}(S)+\operatorname{dim} S ;
$$

it is equal to zero if and only if $S$ is complete intersection; see [21, 2.3.3(b)].

Proposition 7.5. When $\varphi$ is q.c.i. at $\mathfrak{n}$ the following inequalities are satisfied:

$$
\operatorname{cmd} S \leq \operatorname{cmd} R \quad \text { and } \quad \operatorname{cid} S \leq \operatorname{cid} R \text {. }
$$

Equalities hold if grade $_{R} S=\operatorname{dim} R-\operatorname{dim} S$; in particular, if $R$ is CohenMacaulay.

Remark. Conjecture 4.2 predicts that equalities always hold: See (7.5.1) and (7.5.2).

Proof. Let $R \stackrel{\dot{\varphi}}{\rightarrow} R^{\prime} \stackrel{\varphi^{\prime}}{\longrightarrow} \widehat{S}$ be a Cohen factorization. The functions cmd and cid are additive on flat extensions by $[21,1.2 .6, \mathrm{~A} .11]$ and $[5,3.6]$, respectively. As they vanish on the regular rings $R^{\prime} / \mathfrak{m} R^{\prime}$ and $\widehat{S} / \mathfrak{n} \widehat{S}$, we see that $R$ and $R^{\prime}$ are Cohen-Macaulay simultaneously, and that we may assume the map $\varphi$ is surjective.

From the definition and formula (4.1.1) we now obtain an equality

$$
\operatorname{cmd} S=\operatorname{cmd} R-\left(\operatorname{dim} R-\operatorname{dim} S-\operatorname{grade}_{R} S\right) .
$$

On the other hand, the definition, (5.3.1), and (1.2.1) yield

$$
\begin{aligned}
\operatorname{cid} S & =\varepsilon_{2}(S)-\varepsilon_{1}(S)+\operatorname{dim} S \\
& =\varepsilon_{2}(R)-\varepsilon_{1}(R)+\operatorname{rank}_{k}(I / \mathfrak{m} I)-\operatorname{rank}_{S} \mathrm{H}_{1}(E)+\operatorname{dim} S \\
& =\operatorname{cid} R-\left(\operatorname{dim} R-\operatorname{dim} S-\operatorname{grade}_{R} S\right) .
\end{aligned}
$$

The desired assertions follow because $\operatorname{dim} R \geq \operatorname{dim} S+\operatorname{grade}_{R} S$ always holds, see [21, p. 413 and 1.2.14], with equality if $R$ is Cohen-Macaulay, see [21, 2.1.4]. 
For surjective maps $\varphi$ the next corollary is proved in [26, Corollary 5].

Corollary 7.6. Assume that $\varphi$ is q.c.i. at $\mathfrak{n}$.

If the ring $R$ is Cohen-Macaulay, then so is $S$.

The ring $R$ is Gorenstein if and only if so is $S$.

Proof. The first assertion is already contained in the theorem. The second one comes from [11, 7.7.2], since $\varphi$ is quasi-Gorenstein at $\mathfrak{n}$ by Proposition 7.4.

The next result can also be obtained from $[8,1.5]$ or 8.6 below.

Proposition 7.7. Any two conditions below imply the third one.

(a) The homomorphism $\varphi$ is q.c.i. at $\mathfrak{n}$.

(b) The ring $R$ is complete intersection.

(c) The ring $S$ is complete intersection.

Proof. As in the proof of Proposition 7.5, we may assume that $\varphi$ is surjective.

When (a) holds, Theorem 5.3 gives $\varepsilon_{3}(R)=\varepsilon_{3}(S)$. Vanishing of $\varepsilon_{3}$ characterizes complete intersections, see $[28,3.5 .1$ (iii)] or $[7,7.3 .3]$, whence (b) $\Longleftrightarrow$ (c).

When (b) and (c) hold, and $\varkappa: Q \rightarrow \widehat{R}$ is a surjective homomorphism with $Q$ regular local, then both ideals $I=\operatorname{Ker} \varkappa$ and $J=\operatorname{Ker}(\widehat{\varphi} \varkappa)$ are generated by regular sequences. By 2.1 and Lemma 1.4, $J \widehat{R}$ is quasi-complete intersection. Now $J \widehat{R}=I \widehat{R}$, so $I$ is quasi-complete intersection by Lemma 1.3 .

\section{HOMOMORPHISMS OF NOETHERIAN RINGS}

In this section $\varphi: R \rightarrow S$ denotes a homomorphism of noetherian rings.

For such homomorphisms we first define the q.c.i. property in terms of localizations, then show how they can be described in terms of vanishing of André-Quillen homology. Various properties of that theory, collected in Appendix A, are heavily used when studying the stability of this class of maps.

Developments here follow the treatment of l.c.i. homomorphisms in $[8, \S 5]$ and proofs that can be transposed with only superficial changes have been omitted. 
For $\mathfrak{q} \in \operatorname{Spec} S$ we let $\mathfrak{q} \cap R$ denote the prime ideal $\varphi^{-1}(\mathfrak{q})$ of $R$. As usual, we set $k(\mathfrak{q})=S_{\mathfrak{q}} / \mathfrak{q} S_{\mathfrak{q}}$ and call $k(\mathfrak{q}) \otimes_{R} S$ the fiber of $\varphi$ at $\mathfrak{q}$. The induced homomorphism of local rings $\varphi_{\mathfrak{q}}: R_{\mathfrak{q} \cap R} \rightarrow S_{\mathfrak{q}}$ is called the localization of $\varphi$ at $\mathfrak{q}$.

8.1. We say that the homomorphism $\varphi$ is quasi-complete intersection (or q.c.i.) if it is q.c.i. at every $\mathfrak{q} \in \operatorname{Spec} S$.

This notion mimics those of locally complete intersection (or l.c.i) homomorphism in [8] and of quasi-Gorenstein homomorphism in [11], defined by the corresponding condition on $\varphi_{\mathfrak{q}}$. Proposition 7.4 clarifies the relationships:

8.2. Hierarchy. The homomorphism $\varphi$ is l.c.i. if and only if it is q.c.i. and the $R$-module $S_{\mathfrak{q}}$ has finite flat dimension for every prime ideal $\mathfrak{q}$ of $S$.

If $\varphi$ is q.c.i., then it is quasi-Gorenstein.

The concept fits properly into Grothendieck's theory of flat maps; see $[27, \S 6]$.

8.3. Flat homomorphisms. When $\varphi$ is flat it is q.c.i. if and only if it is l.c.i., if and only if the ring $S \otimes_{R} k(\mathfrak{q})$ is locally complete intersection for every $\mathfrak{q} \in \operatorname{Supp} S$.

Proof. The first equivalence holds because for flat homomorphisms the q.c.i. and l.c.i. conditions coincide, by 8.2. The second equivalence is $[8,5.2]$.

Next we describe q.c.i. homomorphisms in terms of André-Quillen homology, by extending from the case of surjective maps the characterization given in [20].

8.5. André-Quillen homology. The homomorphism $\varphi$ is q.c.i. at $\mathfrak{q} \in \operatorname{Spec} S$ if and only if $\mathrm{D}_{n}(S \mid R, k(\mathfrak{q}))=0$ holds for $n \geq 3$.

The homomorphism $\varphi$ is q.c.i. if and only if $\mathrm{D}_{n}(S \mid R,-)=0$ holds for $n \geq 3$.

Proof. Let $R_{\mathfrak{q} \cap R} \rightarrow R^{\prime} \rightarrow \widehat{S_{\mathfrak{q}}}$ be a Cohen factorisation. The kernel of $R^{\prime} \rightarrow \widehat{S_{\mathfrak{q}}}$ is quasi complete intersection if and only if $\mathrm{D}_{n}\left(\widehat{S_{\mathfrak{q}}} \mid R^{\prime}, k(\mathfrak{q})\right)=0$ for $n \geq 3$; see 1.7. By A.2.2, this is equivalent to $\mathrm{D}_{n}(S \mid R, k(\mathfrak{q}))=0$ for $n \geq 3$, whence the first assertion.

The second assertion follows from the first one by [1, S.29].

The next result can be viewed as a sharper version of Proposition 7.7. For surjective $\varphi$ it is due to André [3, Theorem and Proposition]. 
8.6. Complete intersections. Any two conditions below imply the third one.

(a) $\mathrm{D}_{3}(S \mid R, k(\mathfrak{q}))=0$.

(b) The ring $R_{\mathfrak{q} \cap R}$ is complete intersection.

(c) The ring $S_{\mathfrak{q}}$ is complete intersection.

When these conditions hold the homomorphism $\varphi$ is q.c.i. at $\mathfrak{q}$.

Proof. By $[8,4.5], S_{\mathfrak{q}}$ is complete intersection if and only it $\mathbb{Z} \rightarrow S_{\mathfrak{q}}$ is c.i. at $\mathfrak{q} S_{\mathfrak{q}}$. The exact sequence (A.1.1) for $\mathbb{Z} \rightarrow R_{\mathfrak{q} \cap R} \rightarrow S_{\mathfrak{q}} \rightarrow k(\mathfrak{q})$ and the criteria for c.i. and for q.c.i. homomorphisms in A.4 and 8.5, respectively, yield the assertions.

The proof of $[8,5.11]$ shows that the following result is a consequence of 8.5 and standard properties of André-Quillen homology with respect to flat base change.

8.7. Flat base change. Let $R^{\prime}$ be a noetherian ring, $\rho: R \rightarrow R^{\prime}$ a homomorphis$m$ of rings such that $S \otimes_{R} R^{\prime}$ is noetherian, and set $\varphi^{\prime}=\varphi \otimes_{R} R^{\prime}: R^{\prime} \rightarrow S \otimes_{R} R^{\prime}$.

(1) If $\varphi$ is q.c.i. and $\operatorname{Tor}_{n}^{R}\left(S, R^{\prime}\right)=0$ holds for all $n \geq 1$, then $\varphi^{\prime}$ is q.c.i.

(2) If $\varphi^{\prime}$ is q.c.i. and $\rho$ is faithfully flat, then $\varphi$ is q.c.i.

The next three items involve also a noetherian $\operatorname{ring} Q$ and a homomorphism of rings $\psi: Q \rightarrow R$. For those assertions that come in two versions it clearly suffices to prove the statement that includes the text in parentheses.

8.8. Composition. If $\varphi$ is q.c.i. (at some $\mathfrak{q} \in \operatorname{Spec} S$ ) and $\psi$ is q.c.i. (at $\mathfrak{q} \cap R$ ), then the composed homomorphism $\varphi \psi$ is q.c.i. (at $\mathfrak{q})$.

Proof. The exact sequence (A.1.1) for $Q \rightarrow R \rightarrow S \rightarrow k(\mathfrak{q})$ yields $\mathrm{D}_{n}(S \mid Q, k(\mathfrak{q}))=$ 0 for $n \geq 3$, due to 8.5. By the same token, $\varphi \psi$ is q.c.i. at $\mathfrak{q}$.

8.9. Decomposition. Assume that $\varphi \psi$ is q.c.i. (at some $\mathfrak{q} \in \operatorname{Spec} S$ ).

(1) If $\psi$ is l.c.i. (at $\mathfrak{q} \cap R)$, then $\varphi$ is q.c.i. (at $\mathfrak{q})$.

(2) If $\varphi$ is q.c.i. (at $\mathfrak{q})$, then $\psi$ is q.c.i. (at $\mathfrak{q} \cap R$ ).

(3) If $\mathrm{fd}_{R} S_{\mathfrak{q}}$ is finite for some $\mathfrak{q} \in \operatorname{Spec} S$, then $\varphi$ is c.i. at $\mathfrak{q}$ and $\psi$ is q.c.i. at $\mathfrak{q} \cap R$. 
Proof. (1) Set $l=k(\mathfrak{q})$. The exact sequence (A.1.1) for $Q \rightarrow R \rightarrow S \rightarrow l$ yields $\mathrm{D}_{n}(S \mid R, l)=0$ for $n \geq 3$ by 8.5 and A.4, so $\varphi$ is q.c.i. at $\mathfrak{q}$ by 8.5 .

(2) The same exact sequence as in (1) here yields $\mathrm{D}_{n}(R \mid Q, k(\mathfrak{q} \cap R))=0$ for $n \geq 3$ by 8.5 , and the latter also shows that $\psi$ is q.c.i. at $\mathfrak{q} \cap R$.

(3) Since $\mathrm{D}_{n}(S \mid Q, l) \rightarrow \mathrm{D}_{n}(S \mid R, l)$ is surjective for $n=4$ if $\operatorname{char}(l) \neq 2$ and for $n=3$ if $\operatorname{char}(l)=2$, see A.3, we get $\mathrm{D}_{4}(S \mid R, l)=0$ if $\operatorname{char}(l) \neq 2$ and $\mathrm{D}_{3}(S \mid R, l)=0$ if $\operatorname{char}(l)=2$ from 8.5. Now $\varphi$ is c.i. at $\mathfrak{q}$ by A.4, so $\psi$ is q.c.i. at $\mathfrak{q} \cap R$ by $(2)$.

8.10. Flat descent. When $\varphi$ is faithfully flat the composed homomorphism $\varphi \psi$ is q.c.i. if and only if $\varphi$ is l.c.i. and $\psi$ is q.c.i.

Proof. The "if" part comes from 8.8. The converse follows from 8.9(3), because faithfully flat homomorphisms induce surjections on spectra.

By 8.3, the following result applies to homomorphisms essentially of finite type.

8.11. Factorizable homomorphisms. Assume $\varphi=\varphi^{\prime} \dot{\varphi}$, where $\dot{\varphi}$ and $\varphi^{\prime}$ are homomorphism of rings such that $\dot{\varphi}$ is l.c.i. and $\varphi^{\prime}$ is surjective.

The homomorphism $\varphi$ is q.c.i. if and only if $\operatorname{Ker}\left(\varphi^{\prime}\right)_{\mathfrak{m}^{\prime}}$ is a quasi-complete intersection ideal of $R_{\mathfrak{m}^{\prime}}^{\prime}$ for every maximal ideal $\mathfrak{m}^{\prime}$ of $R^{\prime} \operatorname{containing} \operatorname{Ker}\left(\varphi^{\prime}\right)$.

Proof. From 8.8 and $8.9(1)$ we see that $\varphi$ is q.c.i. if and only if $\varphi^{\prime}$ is. By Remark 7.3 the latter holds if and only if the ideal $\operatorname{Ker}\left(\varphi^{\prime}\right)_{\mathfrak{p}^{\prime}}$ of $R_{\mathfrak{p}^{\prime}}^{\prime}$ is quasi-complete intersection for every prime ideal $\mathfrak{p}^{\prime}$ of $R^{\prime}$ that contains $\operatorname{Ker}\left(\varphi^{\prime}\right)$. Lemma 1.3 implies that this can be verified by checking only those $\mathfrak{p}^{\prime}$ that are maximal.

For homomorphisms covered by 8.11 the q.c.i. property localizes, in the sense that if it holds at the maximal ideals of $S$, then it does at all prime ideals. In general, localization may fail. Indeed, recall that the non-zero fiber rings of the completion maps $R \rightarrow \widehat{R_{\mathfrak{m}}}$, when $\mathfrak{m}$ ranges over the maximal ideals of $R$, are called the formal fibers of the ring $R$. Ferrand and Raynaud [24, 3.2(i)] give a local domain $R$ with $\widehat{R} \otimes k(0)$ not Gorenstein; thus, $R \rightarrow \widehat{R}$ is q.c.i. at $\mathfrak{m}$ but not q.c.i., see 8.3 .

In our second result concerning localization the hypotheses are placed on $R$, rather than on $\varphi$. It applies, for instance, to excellent rings (as their formal fibers 
are regular) and to homomorphic images of l.c.i. rings (see [10, Main Theorem (b)]).

8.12. Localization. Assume that $R_{\mathfrak{q} \cap R}$ has l.c.i. formal fibers for each $\mathfrak{q}$ in Spec $S$.

When $\varphi$ is q.c.i. at the maximal ideals of $S$, it is q.c.i. and $S$ has l.c.i.formal fibers.

Proof. Choose $\mathfrak{q} \in \operatorname{Spec} S$, a maximal ideal $\mathfrak{n}$ of $S$ with $\mathfrak{n} \supseteq \mathfrak{q}$, and set $\mathfrak{m}=\mathfrak{n} \cap R$. Set $R^{*}=\widehat{R_{\mathfrak{m}}}$ and $S^{*}=\widehat{S_{\mathfrak{n}}}$, and let $R^{*} \rightarrow R^{\prime} \rightarrow S^{*}$ be a Cohen factorization of $\varphi^{*}=\widehat{\varphi_{\mathfrak{n}}}: R^{*} \rightarrow S^{*}$. Choose $\mathfrak{q}^{*} \in \operatorname{Spec} S^{*}$ with $\mathfrak{q}^{*} \cap S=\mathfrak{q}$, and set $\mathfrak{p}^{\prime}=\mathfrak{q}^{*} \cap R^{\prime}$.

The map $R^{*} \rightarrow R^{\prime}$ is c.i. at $\mathfrak{p}^{\prime}$ as it is flat, $R^{*}$ and $R^{\prime}$ are complete, and $R^{\prime} / \mathfrak{m} R^{\prime}$ is regular; see $\left[10, \S 3\right.$, Step 1]. By A.4 this gives $\mathrm{D}_{n}\left(R^{\prime} \mid R^{*}, k\left(\mathfrak{p}^{\prime}\right)\right)=0$ for $n \geq 2$.

In addition, $R_{\mathfrak{m}} \rightarrow R^{\prime} \rightarrow S^{*}$ is a Cohen factorization of $\varphi_{\mathfrak{n}}$, so $\varphi^{*}$ is q.c.i. at $\mathfrak{n} S^{*}$ by Lemma 7.2 , hence $\mathrm{D}_{n}\left(S^{*} \mid R^{\prime}, k\left(\mathfrak{q}^{*}\right)\right)=0$ for $n \geq 3$ by 8.5 . The exact sequence (A.1.1) for $R^{*} \rightarrow R^{\prime} \rightarrow S^{*} \rightarrow k\left(\mathfrak{q}^{*}\right)$ now yields $\mathrm{D}_{n}\left(S^{*} \mid R^{*}, k\left(\mathfrak{q}^{*}\right)\right)=0$ for $n \geq 3$.

As $R_{\mathfrak{m}}$ has 1.c.i. formal fibers, A.4 gives $\mathrm{D}_{n}\left(R^{*} \mid R, k\left(\mathfrak{q}^{*}\right)\right)$ for $n \geq 2$. The sequence (A.1.1) defined by $R \rightarrow R^{*} \rightarrow S^{*} \rightarrow k\left(\mathfrak{q}^{*}\right)$ gives $\mathrm{D}_{n}\left(S^{*} \mid R, k\left(\mathfrak{q}^{*}\right)\right)=0$ for $n \geq 3$, so the composed map $R \rightarrow S \rightarrow S^{*}$ is q.c.i. at $\mathfrak{q}^{*}$ by 8.5. Since $S \rightarrow S^{*}$ is flat, $8.9(3)$ shows that it is c.i. at $\mathfrak{q}^{*}$ and $R \rightarrow S$ is q.c.i. at $\mathfrak{q}$.

We obtain the desired assertions by varying the choices of $\mathfrak{q}, \mathfrak{n}$, and $\mathfrak{q}^{*}$.

The proof of $[9,6.11]$ shows that 8.12 and 8.7 imply the next property.

8.13. Completion. Assume that $R_{\mathfrak{q} \cap R}$ has l.c.i. formal fibers for each $\mathfrak{q} \in$ Spec $S$.

Let $I \subseteq R$ and $J \subsetneq S$ be ideals, such that $\varphi(I) \subseteq J$, and let $\varphi^{*}: R^{*} \rightarrow S^{*}$ be the induced map of the corresponding ideal-adic completions.

(1) If $\varphi$ is q.c.i., then so is $\varphi^{*}$.

(2) If $I$ is contained in the Jacobson radical of $R$ and $\varphi^{*}$ is q.c.i., then so is $\varphi$. 


\section{Appendix A. André-Quillen homology}

For each $A$-algebra $B$ and every $B$-module $N$, André [1] and Quillen [34] constructed homology groups $\mathrm{D}_{n}(B \mid A, N)$ with remarkable functorial properties. A few results crucial for this paper, taken from [1] and [8], are collected below.

Let $A \stackrel{\alpha}{\rightarrow} B \stackrel{\beta}{\rightarrow} C \stackrel{\gamma}{\rightarrow} l$ be homomorphisms of noetherian rings, where $l$ is a field.

A.1. For $k=k(\operatorname{Ker} \gamma)$ and each $n \geq 0$ there is a natural exact sequence of l-modules

(A.1.1) $\mathrm{D}_{n}(B \mid A, k) \otimes_{k} l \longrightarrow \mathrm{D}_{n}(C \mid A, l) \longrightarrow \mathrm{D}_{n}(C \mid B, l) \stackrel{\partial_{n}}{\longrightarrow} \mathrm{D}_{n-1}(B \mid A, k) \otimes_{k} l$.

Indeed, $\alpha$ and $\beta$ define a Jacobi-Zariski exact sequence with coefficients in $l$, see $[1,5.1]$, which differs from A.1.1 only because in it $\mathrm{D}_{n}(B \mid A, l)$ appears in place of $\mathrm{D}_{n}(B \mid A, k) \otimes_{k} l$. However, these modules are isomorphic by $[1,4.58]$.

For $\mathfrak{q} \in \operatorname{Spec} C$ let $\widehat{C_{\mathfrak{q}}}$ denote the $\mathfrak{q} C_{\mathfrak{q}}$-adic completion of $C_{\mathfrak{q}}$ and set $\mathfrak{p}=\mathfrak{q} \cap B$. A.2. By [1, 4.58 and 5.27] there are natural isomorphisms

$$
\mathrm{D}_{n}(C \mid B, k(\mathfrak{q})) \cong \mathrm{D}_{n}\left(C_{\mathfrak{q}} \mid B, k(\mathfrak{q})\right) \cong \mathrm{D}_{n}\left(C_{\mathfrak{q}} \mid B_{\mathfrak{p}}, k(\mathfrak{q})\right) \quad \text { for } \quad n \geq 0
$$

If $B_{\mathfrak{p}} \rightarrow B^{\prime} \rightarrow \widehat{C_{\mathfrak{q}}}$ is a Cohen factorization of $\beta_{\mathfrak{q}}$, then (A.2.1) and [5, 1.7] give

$$
\mathrm{D}_{n}(C \mid B, k(\mathfrak{q})) \cong \mathrm{D}_{n}\left(\widehat{C_{\mathfrak{q}}} \mid B^{\prime}, k(\mathfrak{q})\right) \quad \text { for } \quad n \geq 2 .
$$

A.3. Set $l=k(\mathfrak{q})$ and let $\gamma: C \rightarrow l$ be the canonical surjection.

If $\operatorname{fd}_{B} C_{\mathfrak{q}}$ is finite, then in (A.1.1) one has $\widetilde{\partial}_{n}=0$ in the following cases:

(a) $n=2 i$ for some integer $i$ with $1 \leq i<\infty$ and $\operatorname{char}(l)=0$.

(b) $n=2 i$ for some integer $i$ with $1 \leq i \leq \operatorname{char}(l)$.

(c) $n=3$ and $\operatorname{char}(l)=2$.

Indeed, in view of (A.2.1) we may assume $B=B_{\mathfrak{p}}, C=C_{\mathfrak{q}}$, and the homomorphisms $\alpha$ and $\beta$ are local. Cases (a) and (b) then are settled by [8, 4.7]. When $\operatorname{char}(l)=2$ the map $\pi_{n}(\alpha): \pi_{n}(A) \otimes_{k} l \rightarrow \pi_{n}(B)$ is injective for all integers $n \geq 2$, not just for the even ones, see [6], so case (c) follows from the proof of $[8,4.7]$

A.4. For $\mathfrak{q} \in \operatorname{Spec} C$ the following conditions are equivalent.

(i) $\beta$ is c.i. at $\mathfrak{q}$. 
(ii) $\mathrm{D}_{\geqslant 2}(C \mid B, k(\mathfrak{q}))=0$.

(iii) $\mathrm{D}_{2}(C \mid B, k(\mathfrak{q}))=0$.

(iv) $\mathrm{D}_{\geqslant q}(C \mid B, k(\mathfrak{q}))=0$ for some some integer $q$, and $\operatorname{fd}_{B} C_{\mathfrak{q}}$ is finite.

(v) $\mathrm{D}_{n}(C \mid B, k(\mathfrak{q}))=0$ for some integer $n$ with $3 \leq n \leq 2 m-1$, where $m$ is an integer such that $(m-1)$ ! is invertible in $k(\mathfrak{q})$, and $\operatorname{fd}_{B} C_{\mathfrak{q}}$ is finite.

This is a consequence of $[8,1.2,1.3$, and 1.4], via (A.2.1).

We finish with two open questions suggested by results in the main text.

A.5. The equivalence of conditions (i) and (ii) in A.4 has a parallel in 8.5. It is natural to ask whether analogs of other conditions hold:

(iii) Does $\mathrm{D}_{3}(C \mid B, k(\mathfrak{q}))=0$ imply that $\beta$ is q.c.i. at $\mathfrak{q}$ ?

(iv) Does $\mathrm{D}_{\geqslant q}(C \mid B, k(\mathfrak{q}))=0$ for some some integer $q$ imply that $\beta$ is q.c.i. at $\mathfrak{q}$ ?

Special cases of (iii) are covered by 8.6. An affirmative answer to (iv) was conjectured by Quillen [34, 5.6] when $\beta$ is of finite type and in [8, p. 459] in general. That conjecture was proved in $[8,1.3]$ in case $C_{\mathfrak{q}}$ has finite flat dimension over $B$, and in $[16$, Theorem 1$]$ in case $\beta$ admits a right inverse ring homomorphism.

\section{ACKNOWLEDGEMENT}

We thank Javier Majadas Soto for pointing us to his work on the subject, and Ray Heitmann, Craig Huneke, and Srikanth Iyengar for useful conversations.

\section{REFERENCES}

[1] M. André, Homologie des algèbres commutatives, Grundlehren Math. Wiss. 204, Springer, Berlin, 1974.

[2] M. André, La $(2 p+1)$-ème déviation d'un anneau local, Enseignement Math. (2) 23 (1977), 239-248.

[3] M. André, Pairs of complete intersections, J. Pure Appl. Algebra 38 (1985), 127-133.

[4] E. F. Assmus, Jr., On the homology of local rings, Illinois J. Math. 3 (1959), 187-199.

[5] L. L. Avramov, Homology of local flat extensions and complete intersection defects, Math. Ann. 228 (1977), 27-37.

[6] L. L. Avramov, Descente des déviations par homomorphismes locaux et génération des idéaux de dimension projective finie, C. R. Acad. Sci. Paris, Sér. I Math. 295 (1982), 665668.

[7] L. L. Avramov, Infinite free resolutions, Six lectures on commutative algebra (Bellaterra, 1996), Progress in Math. 166, Birkhäuser, Basel, 1998; 1-118. 
[8] L. L. Avramov, Locally complete intersection homomorphisms and a conjecture of Quillen on the vanishing of cotangent homology, Ann. of Math. (2) 150 (1999), 455-487.

[9] L. L. Avramov, H.-B. Foxby, Locally Gorenstein homomorphisms, Amer. J. Math. 114 (1992), 1007-1047.

[10] L. L. Avramov, H.-B. Foxby, Grothendieck's localization problem, Commutative Algebra: Syzygies, Multiplicities, and Birational Algebra (South Hadley, 1992), Contemp. Math. 159, Amer. Math. Soc., Providence, RI, 1994; 1-13.

[11] L. L. Avramov, H.-B. Foxby, Ring homomorphisms and finite Gorenstein dimension, Proc. London Math. Soc. (3) 75 (1997), 241-270.

[12] L. L. Avramov, H.-B. Foxby, Cohen-Macaulay properties of ring homomorphisms, Adv. Math. 133 (1998), 54-95.

[13] L. L. Avramov, H.-B. Foxby, B. Herzog, Structure of local homomorphisms, J. Algebra 164 (1994), 124-145.

[14] L. L. Avramov, N. V. Gasharov, I. P. Peeva, A periodic module of infinite virtual projective dimension, J. Pure Appl. Algebra 65 (1989), 1-5.

[15] L. L. Avramov, N. V. Gasharov, I. P. Peeva, Complete intersection dimension, Publ. Math. I.H.E.S. 86 (1997), 67-114.

[16] L. L. Avramov, S. B. Iyengar, André-Quillen homology of algebra retracts, Ann. Sci. École Normale Sup. (4) 36 (2003), 431-462.

[17] L. L. Avramov, S. B. Iyengar, Gorenstein algebras and Hochschild cohomology, Michigan Math. J. 57 (2008), 17-35.

[18] L. L. Avramov, L. C. Sun, Cohomology operators defined by a deformation. J. Algebra 204 (1998), 684-710.

[19] A. Blanco, J. Majadas, A. G. Rodicio, Projective exterior Koszul homology and decomposition of the Tor functor, Invent. Math. 123 (1996), 123-140.

[20] A. Blanco, J. Majadas, A. G. Rodicio, On the acyclicity of the Tate complex, J. Pure Appl. Algebra 131 (1998), 125-132.

[21] W. Bruns, J. Herzog, Cohen-Macaulay rings. Revised edition, Adv. Studies Math. 39, Cambridge Univ. Press, Cambridge, 1998.

[22] A. Conca, Gröbner bases for spaces of quadrics of low codimension, Adv. Appl. Math. 24 (2000), 111-124.

[23] A. Conca, M.-E. Rossi, G. Valla, Gröbner flags and Gorenstein algebras, Compos. Math. 129 (2001), 95-121.

[24] D. Ferrand, M. Raynaud, Fibres formelles d'un anneau local noethérien, Ann. Sci. École Normale Sup. (4) 3 (1970), 295-311.

[25] L. Fouli, C. Huneke, What is a system of parameters? Proc. Amer. Math. Soc. 139 (2011), 2681-2696.

[26] A. Garcia R., J. J. M. Soto, Ascent and descent of Gorenstein property, Glasgow Math. J. 46 (2004), 205-210.

[27] A. Grothendieck, Éléments de géométrie algébrique, IV. Seconde Partie, Étude locale des schémas et des morphismes de schémas, Publ. Math. I.H.E.S. 24 (1965). 
[28] T. H. Gulliksen, G. Levin, Homology of local rings, Queen's Papers Pure Appl. Math. 20, Queen's Univ., Kingston, ON (1969).

[29] I. B. Henriques, L. M. Şega, Free resolutions over short Gorenstein local rings, Math. Z. 267 (2011), 645-663.

[30] R. Kiełpiński, D. Simson, A. Tyc, Exact sequences of pairs in commutative rings, Fund. Math. 99 (1978), 113-121.

[31] A. R. Kustin, L. M. Şega, A. Vraciu, Minimal quasi-complete intersection ideals, arXiv:1309.1186.

[32] G. Levin, Large homomorphisms of local rings, Math. Scand. 46 (1980), 209-215.

[33] M. Nagata, Local rings, Interscience Tracts Pure Appl. Math. 13, Wiley, New York, 1962.

[34] D. Quillen, On the (co-)homology of commutative rings, Applications of Categorical Algebra (New York, 1968), Proc. Symposia Pure Math. 17, Amer. Math. Soc., Providence, R.I., 1970; 65-87.

[35] A. G. Rodicio, On the free character of the first Koszul homology module, J. Pure Appl. Algebra 80 (1992), 59-64.

[36] A. G. Rodicio, Flat exterior Tor algebras and cotangent complexes, Comment. Math. Helvetici 70 (1995) 546-557; Erratum, Comment. Math. Helvetici 71 (1996), 338.

[37] G. Scheja, Über die Bettizahlen lokaler Ringe, Math. Ann. 155 (1964), 155-172.

[38] J. Shamash, The Poincaré series of a local ring, J. Algebra 12 (1969), 457-470.

[39] J. J. M. Soto, Finite complete intersection dimension and vanishing of André-Quillen homology, J. Pure Appl. Algebra 146 (2000), 197-2007.

[40] J. J. M. Soto, Gorenstein quotients by principal ideals of free Koszul homology, Glasgow Math. J. 42 (2000), 51-54.

[41] J. Tate, Homology of Noetherian rings and of local rings, Illinois J. Math. 1 (1957), 14-27.

Luchezar L. Avramov

Department of Mathematics

University of Nebraska

Lincoln, NE 68588, U.S.A.

E-mail: avramov@math.unl.edu

Inês Bonacho Dos Anjos Henriques

Department of Mathematics

University of Nebraska

Lincoln, NE 68588, U.S.A.

Current address: Department of Pure Mathematics

University of Sheffield

Hicks Building, S3 7RH, U.K. 
E-mail: i.henriques@sheffield.ac.uk

Liana M. Şega

Department of Mathematics and Statistics

University of Missouri

Kansas City, MO 64110, U.S.A.

E-mail: segal@umkc.edu 